\title{
The large-activation-energy analysis of extinction of counterflow diffusion flames with non-unity Lewis numbers of the fuel
}

\author{
Amable Liñána, Daniel Martínez-Ruiz ${ }^{b}$, Marcos Vera $^{\mathrm{b}}$, Antonio L. Sánchez ${ }^{\text {c,* }}$
}

\begin{abstract}
A B S T R A C T
Large-activation-energy asymptotic techniques are used to describe the effects of non-unity Lewis numbers of the fuel on strain-induced extinction of axisymmetric counterflow diffusion flames. The present work extends and clarifies previous investigations by accounting also for variable density and variable transport properties of the gas. In our asymptotic analysis the flame structure near extinction is, at leading order, given by the Burke-Schumann limit of infinitely fast reaction; i.e. two outer regions of equilibrium flow, with the fuel and the oxygen separated by an infinitesimally thin reaction layer where they arrive by diffusion in stoichiometric proportions. The leading-order description provides the basic flow structure, including the flame-sheet location, the fuel-consumption rate, the temperature gradients on both sides of the flame, and the peak value of the temperature, which plays a dominant role in flame extinction and differs significantly from the adiabatic-flame value for non-unity lewis numbers. In the near-extinction regime small departures, due to finite rates, from the fast-reaction limit are enough to dominate the structure of the reaction layer, and must be taken into account in this thin layer and in the outer chemically frozen regions. where the corrections are associated with the reactants leaking. with small mass fractions, through the flame. The main effect of the differential diffusion in the nearextinction regime is due to the strong modification of the reaction rates resulting from the changes in the Burke-Schumann peak temperature, with only moderate corrections due to leakage of the reactants through the flame. For large values of the overall stoichiometric ratio $S$ of the diffusion flame, defined as the mass of the air stream needed to burn to completion the unit mass of the fuel stream, the extinction conditions occur in a premixed-flame regime, in which the reaction layer is displaced towards the fuel side with respect to the Burke-Schumann flame sheet position and a fraction of the arriving fuel mass flux leaks through the reaction layer, while the mass fraction of the leaking oxygen decreases to negligibly small values. The asymptotic predictions are tested by comparison with numerical integrations of extinction curves based on continuation methods.
\end{abstract}

\section{Introduction}

Norbert Peters's work had a profound effect on our understanding of many different aspects of Combustion Science. Of paramount importance are his contributions to reduced-kinetic mechanisms, and to the development of flamelet-modeling approaches for the description of finite-tate effects in turbulent combustion [1]. In his famous 1984 review paper [2], he explained in an exemplarily pedagogic manner the role of laminar diffusion flamelets in turbulent non-premixed combustion, building on ideas introduced earlier by Forman Williams [3]. As stated by Norbert Peters [2], "the steady laminar counterflow diffusion flame exhibits a very similar scalar structure as unsteady distorted mixing layers in a turbulent flow field", which leads him to propose the counterflow geometry as "the most representative steady flow field to study chemistry models and molecular transport effects in laminar flamelets". In the present paper, we pay homage to Norber Peters by revisiting the counterflow diffusion flame, with particular attention given to differential-diffusion effects on flame extinction.

The counterflow configuration has been extensively used to analyze the effects of flow strain on diffusion flames [4]. The typical experimental arrangement involves two opposing axisymmetric streams. For the high Reynolds numbers of the experiments, the flow is inviscid in the first approximation outside a thin mixing layer separating the inviscid streams. Near the central stagnation 
point the mixing layer is selfsimilar, with the temperature and species mass fractions varying only with the distance to the stagnation plane. The solution depends on the stagnation-point value of the strain rate imposed on the mixing layer, given by the rate of growth of the radial velocity, constant in each of the external streams.

In diffusion flames, when the rate-controlling chemical reactions have a strong temperature dependence, the nonlinear interaction of the flow and the chemical kinetics leads to the existence of distinguished regimes of ignition and extinction, described by Liñán [5] for unity values of the Lewis numbers of the reactants, consumed by a single irreversible reaction having an Arrhenius rate with large activation energy. While in fuel-air systems that approximation is sufficiently accurate for oxygen, most hydrocarbon molecules are large, and their diffusivities, although of the order of the thermal diffusivity of the gas mixture, are smaller, with values of the fuel Lewis number $L_{\mathrm{F}}$ moderately larger than unity, the approximation $L_{\mathrm{F}}=1$ being reasonably accurate only for methane. On the other hand, hydrogen molecules are small, and the associated Lewis number is $L_{\mathrm{F}} \simeq 0.3$; although an overall reaction is only applicable near the lean flammability limit when the hydrogen is diluted significantly in its feed stream [6]. Both moderately large and moderately small values of $L_{\mathrm{F}}$ are of interest in applications, thereby motivating the present investigation of differentialdiffusion effects that, as explained below, complements many of the previous efforts to analyze extinction.

Liñán's asymptotic analysis [5] showed that the structure of the diffusion flame in near-extinction conditions is given, at leading order, by the Burke-Schumann fast reaction limit, when the reaction layer appears as a flame sheet separating a region without fuel from a region without oxidizer. The description of extinction requires consideration of nonequilibrium effects within the reaction layer. The asymptotic formulation for large activation energies requires a double-limit expansion in which the preexponential factor and the activation energy of the Arrhenius reaction-rate constant both grow to large values in such a way that the resulting temperature decrement in the reaction layer from the peak BurkeSchumann value is small, of the order of the Frank-Kamenetskii temperature, sufficient to decrease the strongly temperature dependent reaction rate by a factor of order unity, and lead eventually to flame extinction. The asymptotic analysis generates, from a reaction-diffusion balance, a canonical boundary-value problem for the reaction-layer structure, whose solution determines the small amount of reactants that leak through the flame in terms of the Damköhler number, the appropriate ratio of the strain time and the reaction time. The leaking reactants, whose growth is responsible for the extinction of the flame, are transported out of the reaction layer, where in the near-extinction regime the reaction is frozen because of the lower temperatures. When the reactant Lewis numbers are equal to unity, as considered in [5], the solution for the reaction-layer structure and the associated computation of the critical extinction conditions are not influenced by the feedback effect of the outer perturbations due to the reactant leakage. For non-unity Lewis numbers of the reactants the inner problem and the first-order corrections to the outer equilibrium solutions are coupled, thereby complicating the determination of the critical extinction conditions, which involves the integration of a set of linearized, chemically frozen transport equations for the leaking reactants in the external streams (see, e.g., the online supplemental appendix of [7]).

It is now known how to deal with the Burke-Schumann (B-S) fast-reaction limit to describe the diffusion-flame-sheet structure for non-unity Lewis numbers [7], and how to calculate the peak flame-sheet temperature, which plays an essential role in determining the finite-rate effects that lead to flame extinction. For unity Lewis numbers of the reactants, in the limit of in- finitely fast reaction, the peak temperature is equal to the adiabatic flame temperature, defined as the combustion temperature of a gas mixture obtained by combining in stoichiometric proportions the air and fuel feed streams with their corresponding initial temperatures. However, for non-unity Lewis numbers the peak temperature of the B-S limit depends on the values of the Lewis numbers, the dilution of the fuel stream, and also on the specific geometric configuration considered, so that, for instance, the resulting peak temperature is typically found to vary along the flame surface. While accounting for differential-diffusion effects in $\mathrm{B}-\mathrm{S}$ computations is a relatively simple task in one-dimensionaltype steady configurations like the counterflow considered here, in time-dependent multi-dimensional flows the computation poses a complicated free-boundary problem, difficult to handle in numerical simulations. As explained in [7-9], these computations can be facilitated for non-unity Lewis numbers by using a formulation based on chemistry-free, Shvab-Zel'dovich, linear combinations of the temperature and reactant mass fractions, not changed directly by the reactions, as conserved scalars.

Different researchers have attempted to solve the extinction problem with non-unity Lewis numbers [10-13], starting with Liñán [10], who addressed also the possibility of extinction in the premixed-flame regime. Extinction in the diffusion-flame regime was studied by Chung and Law [11] for various one-dimensional configurations in the constant-density approximation. Their analysis, which correctly accounts for departures of the flame temperature from the adiabatic value, showed how the reaction-layer structure is reducible to the canonical problem derived originally by Liñan [5] for the equidiffusional case, although in this case the matching procedure involves two terms in the expansions for the outer regions, to include the feedback effect of the leakage of reactants through the flame. This aspect of the problem was also subsequently acknowledged by Kim and Williams [12], who specifically considered equal diffusivities of the two reactants in their counterflow extinction investigation. The validity of a previous attempt [13] to describe differential-diffusion effects on extinction without accounting for these outer perturbations and for departures of peak temperatures from the adiabatic value is necessarily restricted to near-unity Lewis number cases.

The purpose of the present paper is to provide a clarifying description of the extinction for large activation energies of counterflow diffusion flames, based on the appropriate double limit for the diffusion-flame extinction regime. Our work accounts for the effects of variable density and variable transport properties. The accuracy of the asymptotic analysis will be assessed through comparisons with results of finite-rate computations employing a onestep Arrhenius model with large but finite activation temperature.

The paper begins by giving in Section 2 the formulation of the counterflow problem, with a one-step Arrhenius reaction, accounting for variable density and variable transport properties. The structure of the diffusion flame in the Burke-Schumann limit of infinitely fast reaction is formulated in Section 3 for a non-unity Lewis number of the fuel, and the resulting problem is solved numerically for relevant values of $L_{\mathrm{F}}$ and of the fuel-feed dilution. Departures from chemical equilibrium leading to flame extinction are considered in Section 4. The simplified description of the nearextinction conditions occurring in the premixed-flame regime for small values of the B-S heat-loss rate to the fuel side is given in Section 5. Finally, concluding remarks are given in Section 6 .

\section{Description of diffusion flames in counterflow mixing layers}

\subsection{Chemistry model}

We give below the equations and boundary conditions for the description of non-premixed flames in counterflow mixing layers 
separating a stream of air from a stream containing a mixture of fuel and inert. The reaction between the fuel and the oxygen of the air will be considered to occur according to the global irreversible step

$\mathrm{F}+s \mathrm{O}_{2} \rightarrow(1+s) \mathrm{P}+q^{\prime}$,

where $s$ and $q^{\prime}$ are the mass of oxygen consumed and the amount of heat released per unit mass of fuel burnt; the products of the reaction are lumped, for simplicity in the presentation, into a single product P. As noted in [7], the resulting values of these parameters differ only by a small amount for saturated hydrocarbons (e.g., $s=(3.52,3.48)$ and $q^{\prime}=(48.10,44.56) \mathrm{MJ} / \mathrm{kg}$ for heptane and dodecane, respectively). It is of interest that, although the associated values are quite different for hydrogen, for which $s=8$ and $q^{\prime} \simeq 120 \mathrm{MJ} / \mathrm{kg}$, the value of the heat release referred to the unit mass of oxygen burnt $q^{\prime} / s \simeq 15 \mathrm{MJ} / \mathrm{kg}$ is very similar to the values $q^{\prime} / s \simeq 13 \mathrm{MJ} / \mathrm{kg}$ found for alkanes.

In the following analysis, the reaction rate will be modeled using the simple Arrhenius expression

$w_{\mathrm{F}}=B^{\prime} \rho^{\prime} e^{-E /\left(R T^{\prime}\right)} Y_{\mathrm{F}} Y_{\mathrm{O}_{2}}$

for the mass of fuel consumed per unit volume per unit time, first order for the fuel and oxygen for simplicity in the presentation. Here, $\rho^{\prime}$ and $T^{\prime}$ represent the density and temperature of the gas mixture and $Y_{\mathrm{F}}$ and $Y_{\mathrm{O}_{2}}$ are the mass fractions of fuel and oxygen. Two rate parameters appear in (2), namely, the preexponential frequency factor $B^{\prime}$ and the activation energy $E$, which defines the activation temperature $T_{a}=E / R$ when divided by the universal gas constant $R$. The Arrhenius exponential is a strongly dependent function of the temperature when $E$ is large compared with the thermal energy $R T$ (i.e. for $T_{a} / T \gg 1$ ). The much smaller temperature dependence of the frequency factor of the overall, nonelementary, reaction is neglected.

It is worth emphasizing here that (1) is not an elementary reaction; it is an overall reaction representing the underlying stoichiometry of the fuel-oxidation process. Consequently, its overall rate, given in (2), does not follow the law of mass action. In applications, the kinetic parameters $B^{\prime}$ and $E$ could be selected to give a good approximation of the stoichiometric premixed-flame structure. A kinetic model of this type, including unity orders for both reactants, has been shown to be successful in describing methane combustion [14], for instance. It would be straightforward to extend the asymptotic analysis given below to reaction rates with non-unity reaction orders, as done for premixed flames in early work [15].

\subsection{Conservation equations and boundary conditions}

We shall consider the steady axisymmetric counterflow diffusion flame established in a steady mixing layer separating opposed nearly inviscid streams of fuel, coming from $z=\infty$, and air, coming from $z=-\infty$. In the stagnation-point region the radial velocities on the air and fuel sides outside the mixing layer grow linearly with the radial distance to the stagnation point $r$ according to $2 v^{\prime}=A_{\mathrm{A}} r$ and $2 v^{\prime}=A_{0} r$. The strain rates on the air and fuel sides $A_{\mathrm{A}}$ and $A_{o}$ are different, their values being related to the fuel-toair density ratio $\rho_{0}^{\prime} / \rho_{\mathrm{A}}^{\prime}$ by the equation $A_{0}=A_{\mathrm{A}} \sqrt{\rho_{\mathrm{A}}^{\prime} / \rho_{0}^{\prime}}$, resulting from the condition of negligible pressure variation across the mixing layer. In the stagnation-point region the reacting flow is selfsimilar, inside and outside the mixing layer. The density $\rho^{\prime}(z)$, the temperature $T^{\prime}(z)$, and the mass fractions $Y_{\mathrm{F}}(z)$ and $Y_{\mathrm{O}_{2}}(z)$ of fuel and oxygen are functions of the distance $z$ to the stagnation plane, as are the axial velocity $u^{\prime}(z)$ and the strain rate $A^{\prime}(z)=2 v^{\prime} / r$, the latter determining the linear growth with $r$ of the radial velocity $v^{\prime}$. The velocity field is coupled with the density through the con- tinuity equation

$\frac{\mathrm{d}}{\mathrm{d} z}\left(\rho^{\prime} u^{\prime}\right)+\rho^{\prime} A^{\prime}=0$.

Since the radial pressure gradient $-\partial p^{\prime} / \partial r=\rho_{A}^{\prime} A_{A}^{2} r / 4=\rho_{0}^{\prime} A_{0}^{2} r / 4$ is uniform, the radial component of the momentum equation takes the form

$\frac{\mathrm{d}}{\mathrm{d} z}\left(\mu \frac{\mathrm{d} A^{\prime}}{\mathrm{d} z}\right)-\rho^{\prime} u^{\prime} \frac{\mathrm{d} A^{\prime}}{\mathrm{d} z}+\frac{1}{2}\left(\rho_{\mathrm{A}}^{\prime} A_{\mathrm{A}}^{2}-\rho^{\prime} A^{\prime 2}\right)=0$,

where $\mu^{\prime}$ is the viscosity of the gas mixture. The conservation equations for reactants are

$\frac{\mathrm{d}}{\mathrm{d} z}\left(\rho^{\prime} D_{\mathrm{F}} \frac{\mathrm{d} Y_{\mathrm{F}}}{\mathrm{d} z}\right)-\rho^{\prime} u^{\prime} \frac{\mathrm{d} Y_{\mathrm{F}}}{\mathrm{d} z}=w_{\mathrm{F}}$

$\frac{\mathrm{d}}{\mathrm{d} z}\left(\rho^{\prime} D_{\mathrm{O}} \frac{\mathrm{d} Y_{\mathrm{O}_{2}}}{\mathrm{~d} z}\right)-\rho^{\prime} u^{\prime} \frac{\mathrm{d} Y_{\mathrm{O}_{2}}}{\mathrm{~d} z}=s w_{\mathrm{F}}$.

A Fickian description is adopted for the species diffusion velocities, with $D_{\mathrm{F}}$ and $D_{\mathrm{O}}$ representing the molecular diffusivities of fuel and oxygen, respectively. The energy conservation equation is

$\frac{\mathrm{d}}{\mathrm{d} z}\left(k \frac{\mathrm{d} T^{\prime}}{\mathrm{d} z}\right)-\rho^{\prime} u^{\prime} c_{p} \frac{\mathrm{d} T^{\prime}}{\mathrm{d} z}=-q^{\prime} w_{\mathrm{F}}$,

where $k$ and $c_{p}$ are the thermal conductivity and the specific heat at constant pressure, the latter assumed to be constant for simplicity in the presentation. Here $w_{\mathrm{F}}$ is the mass consumption of the fuel per unit volume per unit time, given by the Arrhenius expression (2), involving an oxygen-consumption rate $s w_{\mathrm{F}}$ and a heat-release rate $q^{\prime} w_{\mathrm{F}}$.

The above equations must be supplemented with the equation of state, which is written in the simplified form

$\rho^{\prime} T^{\prime}=\rho_{\mathrm{A}}^{\prime} T_{\mathrm{A}}^{\prime}$,

neglecting the variations of the pressure, an approximation justified for low-Mach-number combustion, and, for simplicity, the variations of the mean molecular mass. Here $T_{\mathrm{A}}^{\prime}$ is the temperature of the air stream. The simple power-law expressions

$\frac{k}{k_{\mathrm{A}}}=\frac{\mu}{\mu_{\mathrm{A}}}=\frac{\rho^{\prime} D_{\mathrm{F}}}{\rho_{\mathrm{A}}^{\prime} D_{\mathrm{F}_{\mathrm{A}}}}=\frac{\rho^{\prime} D_{\mathrm{O}}}{\rho_{\mathrm{A}}^{\prime} D_{\mathrm{O}_{\mathrm{A}}}}=\left(\frac{T^{\prime}}{T_{\mathrm{A}}^{\prime}}\right)^{\sigma}$

will be adopted for the temperature variation of the transport properties from their air-side values, with the exponent $\sigma=0.7$ used in the computations below.

The above equations must be integrated with the boundary conditions

$\left\{\begin{array}{lll}A^{\prime}-A_{0}=Y_{\mathrm{F}}-Y_{\mathrm{F}_{0}}=Y_{\mathrm{O}_{2}}=T^{\prime}-T_{0}^{\prime}=0 & \text { as } & z \rightarrow+\infty \\ A^{\prime}-A_{\mathrm{A}}=Y_{\mathrm{F}}=Y_{\mathrm{O}_{2}}-Y_{\mathrm{O}_{2} \mathrm{~A}}=T^{\prime}-T_{\mathrm{A}}^{\prime}=0 & \text { as } & z \rightarrow-\infty\end{array}\right.$.

where $T_{0}^{\prime}$ represents the fuel-stream temperature and $Y_{\mathrm{F}_{0}}$ and $Y_{\mathrm{O}_{2} \mathrm{~A}} \simeq 0.232$ are the values of the mass fractions of fuel and oxygen in their respective feed streams. The translational invariance present in the counterflow mixing-layer problem is removed by selecting the origin for the transverse coordinate at the stagnation plane, so that $u^{\prime}(0)=0$.

The equations will be written in dimensionless form by introducing the scaled variables

$$
\begin{aligned}
A & =\frac{A^{\prime}}{A_{\mathrm{A}}}, F=-\frac{\rho^{\prime} u^{\prime}}{\rho_{\mathrm{A}}^{\prime}\left(D_{T_{\mathrm{A}}} A_{\mathrm{A}}\right)^{1 / 2}}, \rho=\frac{\rho^{\prime}}{\rho_{\mathrm{A}}^{\prime}}, T=\frac{T^{\prime}}{T_{\mathrm{A}}^{\prime}}, \\
\hat{Y}_{\mathrm{F}} & =\frac{Y_{\mathrm{F}}}{Y_{\mathrm{F}_{0}}}, \hat{Y}_{\mathrm{O}}=\frac{Y_{\mathrm{O}_{2}}}{Y_{\mathrm{O}_{2} \mathrm{~A}}},
\end{aligned}
$$


where $D_{T_{A}}=k_{A} /\left(\rho_{A}^{\prime} c_{p}\right)$ is the air-side value of the thermal diffusivity. The effects of the large variations of density and transport properties occurring across the mixing layer, as a result of the combustion process, can be conveniently accounted for in the formulation by defining a dimensionless heat-conduction-weighted transverse coordinate

$\eta=\left(\frac{A_{\mathrm{A}}}{D_{T_{A}}}\right)^{1 / 2} \int_{0}^{z} T^{-\sigma} \mathrm{d} z$,

incorporating the temperature dependence of the transport properties given in (9). In (12) $z$ appears divided by $\left(D_{T_{A}} / A_{A}\right)^{1 / 2}$, the characteristic mixing-layer thickness based on the constant heat conductivity and density values of the air stream. This alternative transverse coordinate $\eta$, which is different from the HowarthDorodnitsyn variable, will be used below to compare with the analytic description given later.

Introduction of the dimensionless variables defined above into (3)-(10) leads to

$\frac{\mathrm{d} F}{\mathrm{~d} \eta}-T^{\sigma-1} A=0$

$\operatorname{Pr} \frac{\mathrm{d}^{2} A}{\mathrm{~d} \eta^{2}}+F \frac{\mathrm{d} A}{\mathrm{~d} \eta}+\frac{T^{\sigma}}{2}\left(1-\frac{A^{2}}{T}\right)=0$

$\frac{1}{L_{\mathrm{F}}} \frac{\mathrm{d}^{2} \hat{Y}_{\mathrm{F}}}{\mathrm{d} \eta^{2}}+F \frac{\mathrm{d} \hat{\mathrm{Y}}_{\mathrm{F}}}{\mathrm{d} \eta}=T^{\sigma-1} \hat{Y}_{\mathrm{F}} \mathrm{Y}_{\mathrm{O}} \frac{B}{A_{\mathrm{A}}} e^{-T_{\alpha} / T}$

$\frac{\mathrm{d}^{2} \hat{Y}_{\mathrm{O}}}{\mathrm{d} \eta^{2}}+F \frac{\mathrm{d} \hat{Y}_{0}}{\mathrm{~d} \eta}=S T^{\sigma-1} \hat{Y}_{\mathrm{F}} \hat{Y}_{O} \frac{B}{A_{\mathrm{A}}} e^{-T_{\sigma} / T}$

$\frac{\mathrm{d}^{2} T}{\mathrm{~d} \eta^{2}}+F \frac{\mathrm{d} T}{\mathrm{~d} \eta}=-q T^{\sigma-1} \hat{Y}_{\mathrm{F}} \hat{\mathrm{Y}}_{\mathrm{O}} \frac{B}{A_{\mathrm{A}}} e^{-T_{\sigma} / T}$,

subject to $F(0)=0$ and the matching conditions with the outer streams

$\left\{\begin{array}{lll}A-\sqrt{T_{0}}=\hat{Y}_{\mathrm{F}}-1=\hat{Y}_{\mathrm{O}}=T-T_{0}=0 & \text { as } & \eta \rightarrow+\infty \\ A-1=\hat{Y}_{\mathrm{F}}=\hat{Y}_{\mathrm{O}}-1=T-1=0 & \text { as } & \eta \rightarrow-\infty .\end{array}\right.$.

Here $T_{0}=T_{0}^{\prime} / T_{\mathrm{A}}^{\prime}$ represents the fuel-to-air temperature ratio, with the fuel-to-air strain-rate ratio given by $A_{0} / A_{\mathrm{A}}=\left(\rho_{\mathrm{A}}^{\prime} / \rho_{0}^{\prime}\right)^{1 / 2}=\sqrt{T_{0}}$, as follows from the equation of state (8). Here, $L_{\mathrm{F}}=\left(k / c_{p}\right) /\left(\rho^{\prime} D_{\mathrm{F}}\right)$ and $\operatorname{Pr}=\mu /\left(k / c_{p}\right)$ are the fuel Lewis number and the Prandtl number, both assumed to be constant but different from unity. A unity value is assumed for the Lewis number of $\mathrm{O}_{2}$, a reasonably good approximation for fuel-air mixtures. Note that, because of the normalization of the reactant mass fractions, a modified frequency factor $B=B^{\prime} Y_{\mathrm{O}_{2}} \mathrm{~A}$ replaces $B^{\prime}$ in the reaction-rate terms in (15)-(17).

Since we have chosen the origin of $\eta$ where the transverse velocity vanishes, the condition $F(0)=0$ has been added when integrating (13)-(17). If desired, the numerical integration determines the displacements $(F-\eta)_{-\infty}$ and $\left(F-T_{0}^{\sigma-1 / 2} \eta\right)_{+\infty}$ of the air and fuel streams. The numerical integrations given below for equal feed temperatures (i.e. $T_{0}=1$ ) revealed that the displacements are very small, as it is also the case of the deviation of $F$ from $\eta$ across the mixing layer. If we use in the conservation Eqs. (15)-(17) the approximation $F=\eta$ then these equations are, aside from the factor $T^{\sigma-1}$ in the reaction terms, identical to those obtained in the thermodiffusive approximation (i.e. with constant density and constant transport properties), thereby simplifying considerably the analysis.

In addition to the parameters $L_{\mathrm{F}}, \operatorname{Pr}$, and $\sigma$ entering in the transport terms, two dimensionless kinetic parameters, namely, the dimensionless frequency factor $\left(B / A_{A}\right)$ and the nondimensional activation temperature $T_{a}=E /\left(R T_{\AA}^{\prime}\right)$, appear in the reaction terms, along with the dimensionless thermochemical parameters $S$ and $q$. The parameter $S=s Y_{\mathrm{F}_{0}} / Y_{\mathrm{O}_{2} \mathrm{~A}}$ is the mass of the air stream needed to burn the fuel contained in the unit mass of the fuel stream, which appears as the ratio of the reaction terms in (16) and (15); its value depends on the mass fractions of the fuel and the oxygen in their feed streams. Although $S$ is a fairly large number in undiluted fuel-air combustion (e.g., $S \simeq 15$ and $S \simeq 34.5$ for dodecaneair and hydrogen-air cases, respectively), values of order unity are also of interest because they are found in combustion systems with reduced values of $Y_{F_{0}}$ resulting from dilution of the fuel stream. The other thermochemical, exothermicity, parameter is the dimensionless heat release per unit mass of fuel $q=q^{\prime} Y_{\mathrm{F}_{0}} /\left(c_{p} T_{\mathrm{A}}^{\prime}\right)$, based on $T_{\mathrm{A}}^{\prime}$ and on the specific heat at constant pressure $c_{p}$, considered in this paper to be constant; $q$ appears as the ratio of the reaction terms in (17) and (15). Notice that $q / S=\left(q^{\prime} / s\right) Y_{\mathrm{O}_{2} \mathrm{~A}} /\left(c_{p} T_{\mathrm{A}}^{\prime}\right)$ is independent of the fuel dilution in its feed stream. The ratio $q /(S+1)=T_{S}-1$ is the dimensionless temperature increment, based on $T_{\mathrm{A}}^{\prime}$, for the adiabatic isobaric combustion of the gas mixture generated by mixing, in stoichiometric proportions, the unit mass of the fuel stream with the mass $S$ of the air, both at the air-side temperature $T_{\mathrm{A}}^{\prime}$.

\subsection{Conserved scalars from coupling functions in diffusion flames}

To facilitate the analysis of the fast-reaction limit it is convenient to replace two of Eqs. (15)-(17) by two chemistry-free conservation equations, obtained by eliminating the reaction terms through appropriate linear combinations. For instance, multiplying (16) by $q / S$ and adding (17) leads to

$\frac{\mathrm{d}^{2}}{\mathrm{~d} \eta^{2}}\left(T+q \hat{Y}_{O} / S\right)+F \frac{\mathrm{d}}{\mathrm{d} \eta}\left(T+q \hat{Y}_{O} / S\right)=0$,

involving a single coupling function $\left(T+q \hat{Y}_{O} / S\right)$, which can be conveniently expressed as an excess thermal and chemical enthalpy $H=T-1+(q / S)\left(\hat{Y}_{0}-1\right)$ (i.e. enthalpy in excess of that in the air stream) that, divided by its value in the fuel stream, defines

$$
\begin{aligned}
\xi & =\frac{T-1+(q / S)\left(\hat{Y}_{O}-1\right)}{T_{0}-1-q / S} \text { or } \\
T-1 & =(q / S)\left(1-\xi-\hat{Y}_{0}\right)+\left(T_{0}-1\right) \xi .
\end{aligned}
$$

The normalized enthalpy $\xi$ is a conserved scalar, not changing in the reaction, with a Lewis number equal to unity, which satisfies the source-free equation

$\frac{\mathrm{d}^{2} \xi}{\mathrm{d} \eta^{2}}+F \frac{\mathrm{d} \xi}{\mathrm{d} \eta}=0$

with the boundary conditions $\xi(-\infty)=0$ and $\xi(\infty)=1$; so that $\xi$ increases monotonically with $\eta$ from 0 to 1 . The first term in the second relation in (20) shows how the temperature grows when the oxygen mass fraction decreases due to the reaction.

The reaction term can also be eliminated by subtracting (16) from (15) times $S$ to give

$$
\frac{\mathrm{d}^{2}}{\mathrm{~d} \eta^{2}}\left(\frac{S \hat{Y}_{\mathrm{F}}}{L_{\mathrm{F}}}-\hat{Y}_{\mathrm{O}}\right)+F \frac{\mathrm{d}}{\mathrm{d} \eta}\left(S \hat{Y}_{\mathrm{F}}-\hat{Y}_{\mathrm{O}}\right)=0 .
$$

Two different linear coupling functions appear above in the convective and diffusion terms when the fuel Lewis number $L_{\mathrm{F}}$ is different from unity. When these coupling functions are normalized with their values in the air and fuel feed streams we obtain a diffusion-weighted mixture fraction

$\tilde{Z}=\frac{S \hat{Y}_{\mathrm{F}} / L_{\mathrm{F}}-\hat{Y}_{\mathrm{O}}+1}{S / L_{\mathrm{F}}+1}$, 
arising from the diffusion terms, in addition to the classical mixture fraction

$Z=\frac{S \hat{Y}_{F}-\hat{Y}_{O}+1}{S+1}$,

derived from the convective terms, so that (22) takes the compact form

$\frac{\mathrm{S} / L_{\mathrm{F}}+1}{\mathrm{~S}+1} \frac{\mathrm{d}^{2} \tilde{Z}}{\mathrm{~d} \eta^{2}}+F \frac{\mathrm{d} Z}{\mathrm{~d} \eta}=0$.

The boundary conditions (18) together with the definitions given in (20), (23), and (24) lead to

$$
\begin{aligned}
Z-1 & =\tilde{Z}-1=0 \text { and } \xi=1 \text { as } \eta \rightarrow \infty \\
Z & =\tilde{Z}=0 \text { and } \xi=0 \text { as } \eta \rightarrow-\infty,
\end{aligned}
$$

to be used as boundary conditions for (21) and (25), the latter involving the unknowns $Z$ and $\tilde{Z}$. These equations are to be integrated together with (13), (14), and one of the equations, e.g. (17), involving the reaction term.

\subsection{Sample computations}

Results of numerical integrations of (14)-(18)-carried out for $\sigma=0.7$ and $P r=0.7$, as is appropriate for fuel-air mixtures-are given in Fig. 1 for dilute fuel feed $(S=2)$ and equal feed temperatures $\left(T_{0}=1\right)$. The figure includes solid curves representing temperature and reactant mass fractions for $L_{\mathrm{F}}=0.3$ (lower plot) and $L_{\mathrm{F}}=2$ (upper plot) along with dashed curves representing results for infinitely fast reaction, to be discussed later. For both flames in Fig. 1 , the values of the ratio $B / A_{\mathrm{A}}$ have been selected using the estimate (45) to place the flame near extinction.

The Lewis numbers are representative of hydrogen $\left(L_{\mathrm{F}}=0.3\right)$ and large hydrocarbon $\left(L_{\mathrm{F}}=2\right)$ molecules, respectively. Because of its higher diffusivity, the hydrogen reaction layer, where the temperature peaks, sits farther from the stagnation plane than that of the hydrocarbon flame. Differential diffusion has a noticeable effect on the resulting temperature increment $T-1$, measured in the plots with the value $q /(S+1)$ corresponding to adiabatic stoichiometric combustion. The temperature profile for $L_{\mathrm{F}}=2$ displays a peak value below the adiabatic flame temperature $T_{S}=$ $1+q /(S+1)$, whereas a superadiabatic peak temperature above $T_{S}$ is found for the diffusivity of hydrogen.

As previously anticipated, for the description of gaseous counterflow mixing layers the heat-conduction-weighted coordinate $\eta$, defined in (12), is more convenient than the commonly used density-weighted coordinate, in that it facilitates the analytic description of the flow. The simplification stems from the fact that with this coordinate the variation with temperature of the heat conductivity does not appear in the transport terms of (14)-(17). There is the additional unexpected advantage that the resulting transverse mass flux $F$ is approximately given by $F=\eta$, holding for all conditions tested in this paper, as illustrated in Fig. 2 by comparing $F=\eta$ with the function $F(\eta)$ given by the numerical computations shown in Fig. 1. Also shown in the plot is the variation with $\eta$ of $\xi, Z, \widetilde{Z}$, and the strain rate $A(\eta)$, or radial velocity scaled with $r$, which has an overshoot due to the pressure gradient imposed on the heated gas of the mixing layer. If this approximation $F=\eta$ is used in (15)-(17) then the temperature and mass fractions become identical, with the coordinate $\eta$, as given by the approximation of constant density and transport properties. The expected degree of accuracy of the resulting approximate description is investigated in Fig. 2 by plotting the overshoot above 1 of the source term of $F$ in (13), and also the excess enthalpy $\xi$ computed numerically, as defined in (20), to compare with the analytic prediction $\xi=\frac{1}{2}[1+\operatorname{erf}(\eta / \sqrt{2})]$ obtained from (21) with $F=\eta$. As can be seen, the resulting departures in $\xi$ are negligibly small, thereby motivating the use of the approximation $F=\eta$ in (15)-(17) to obtain analytic predictions for different flame properties.

\section{The B-S limit of infinitely fast reaction}

\subsection{General considerations}

As mentioned above, in computing the reactive mixing layer, one can replace two of the three Eqs. (15)-(17) by the chemistryfree Eqs. (21) and (25). This replacement is required in the B$\mathrm{S}$ limit, $B / A_{\mathrm{A}} \rightarrow \infty$, of infinitely fast reaction rates, for which the dimensionless reaction rates on the right-hand side of (15)-(17), which involve the ratio $B / A_{\mathrm{A}}$ as a factor, lead to the same chemical-equilibrium condition of non-coexistence

$\hat{Y}_{\mathrm{F}} \hat{Y}_{\mathrm{O}}=0$

of the reactants, outside an infinitesimally thin reaction layer located at $\eta=\eta_{f}$, because the fast reaction prevents them from crossing the reaction layer. The B-S fast-reaction problem is then reduced to the integration of the reaction-free Eqs. (13), (14), (21) and (25), together with (27), which corresponds to the complete consumption of either the fuel or the oxygen outside the flame. In the B-S limit $B / A_{\mathrm{A}} \rightarrow \infty$ the reaction terms in (15)-(17) become Dirac-delta source terms, that-necessarily balanced by the diffusion terms-act as sinks for the reactants-which in this limit arrive at the flame sheet with zero concentration-and as a heat source for the temperature, which peaks at $\eta=\eta_{f}$ with a value $T=T_{f}$. This, as well as the value $\xi_{f}$ of $\xi$ at the flame, are to be calculated as part of the solution. The fuel and the oxygen reach the flame sheet by diffusion from opposite sides to arrive in stoichiometric proportions, with the ratio $1 / S$ of the Dirac-delta type reaction terms in (15) and (16). The associated mass fraction gradients, which jump down to zero across the reaction layer, are defined by

$m_{\mathrm{F}}=\left.\frac{1}{L_{\mathrm{F}}} \frac{\mathrm{d} \hat{Y}_{\mathrm{F}}}{\mathrm{d} \eta}\right|_{\eta_{f}^{+}}=-\left.\frac{1}{\mathrm{~S}} \frac{\mathrm{d} \hat{Y}_{\mathrm{O}}}{\mathrm{d} \eta}\right|_{\eta_{f}^{-}}=\frac{m_{\mathrm{O}}}{\mathrm{S}}$.

Here $m_{\mathrm{F}}$ and $m_{\mathrm{O}}=S m_{\mathrm{F}}$ are appropriate nondimensional measures of the fuel and oxygen flame consumption rates. For example, the value of $m_{\mathrm{F}}$ can be used to evaluate the rate of fuel consumption per unit flame surface $m_{\mathrm{F}}^{\prime \prime}=\rho^{\prime} D_{\mathrm{F}} \mathrm{d} Y_{\mathrm{F}} / \mathrm{d} z$, giving

$\frac{m_{\mathrm{F}}^{\prime \prime}}{Y_{\mathrm{F}_{0}} \rho_{\mathrm{A}}^{\prime} \sqrt{D_{T_{A}} A_{\mathrm{A}}}}=m_{\mathrm{F}}$.

The heat released at the flame, given by $q m_{\mathrm{F}}=(q / S) m_{O}$, is transported by conduction towards both sides of the flame according to

$q m_{\mathrm{F}}=\left.\frac{\mathrm{d} T}{\mathrm{~d} \eta}\right|_{\eta_{f}^{-}}-\left.\frac{\mathrm{d} T}{\mathrm{~d} \eta}\right|_{\eta_{f}^{+}}$

because the Dirac reaction term in (17) is $q$ times the reaction term in (15). The relations (28) and (30) can be obtained directly from the reaction-free conservation Eqs. (19) and (22), because in the thin reaction layer the two infinitely large second derivatives in their first term must compensate each other with negligible effect of the convective term.

For the counterflow problem, and also for other onedimensional problems, the flame location $\eta_{f}$ and the peak temperature at the flame $T_{f}$ can be determined by patching two reaction free boundary-value problems, in which (13)-(15) and (17) are integrated for $\eta>\eta_{f}$ (where $\hat{Y}_{0}=0$ ), while (13), (14), (16), and (17) are integrated for $\eta<\eta_{f}$ (where $\left.\hat{Y}_{\mathrm{F}}=0\right)$. At the flame sheet, we use the condition $\hat{Y}_{\mathrm{F}}=\hat{Y}_{\mathrm{O}}=0$ resulting from the limiting fastreaction-rate condition (27) along with the conditions of continuity of $T, F, F^{\prime}$, and $F^{\prime \prime}$. The solution is uniquely determined with the additional relations (28) and (30). These equations relate the jumps 

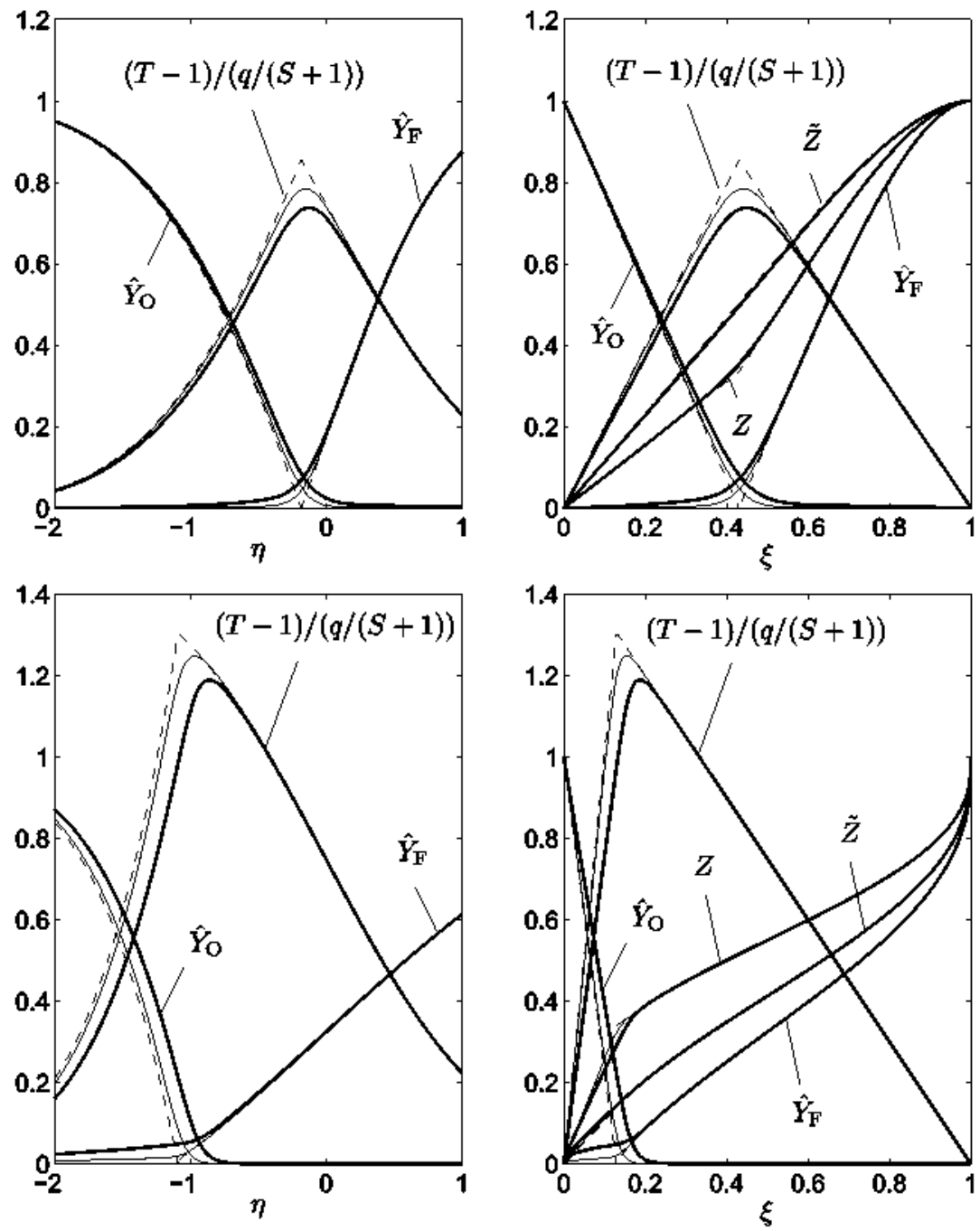

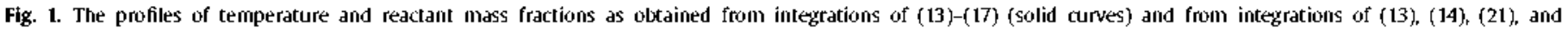

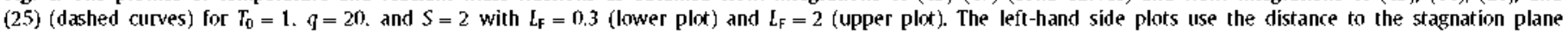

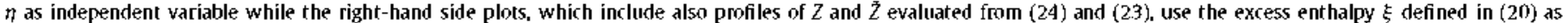

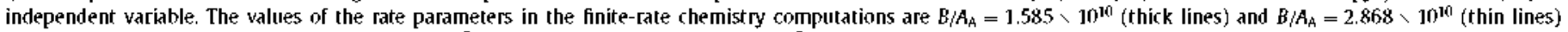

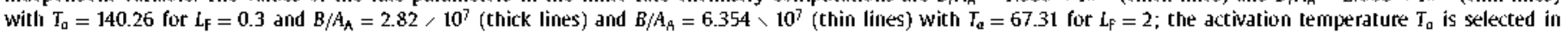
both computations to give a value of the Zel'dovich number based on the B-S peak temperature $\beta=10$, with $\beta$ defined in (44),

in the gradients of temperature and reactant mass fractions at the flame sheet, resulting from the Dirac-delta nature of the chemicalreaction term in the limit $B / A_{A} \rightarrow \infty$.

\subsection{Conserved-scalar description of the fast-reaction limit}

Handling for non-unity Lewis numbers the B-S limit of infinitely fast reaction becomes considerably more difficult in unsteady or multidimensional situations, for which the determination of the flame location poses a complicated free-boundary problem. For those general configurations, the velocity, temperature, and fuel and oxygen concentrations can be obtained (see [7-9]) using the fast-reaction limiting condition (27), i.e. $\hat{Y}_{0} \hat{Y}_{F}=0$, together with the coupling functions $\xi, Z$, and $\hat{Z}$ defined in (20), (23), and (24), which satisfy chemistry-free transport equations (given for the counterflow in (21) and (25)), to be integrated together with the continuity and momentum equation, needed to determine the accompanying velocity field. To solve these equations we need to relate $T$ and $Z$ with $\xi$ and $\mathcal{Z}$. These relations can be obtained using the fast-reaction condition (27) of non-coexistence of $\hat{Y}_{0}$ and $\hat{Y}_{F}$, which are simultaneously zero at the flame, given by the iso-surface $Z=Z_{S}=1 /(1+S)$, or $\tilde{Z}=\tilde{Z}_{S}=1 /\left(1+S / L_{F}\right)$, as follows from the definitions (23) and (24). For $\hat{Z} \geq \hat{Z}_{S}$

$$
\begin{aligned}
\hat{Y}_{0} & =0 \text { and } \hat{Y}_{\mathrm{F}}=\frac{Z-Z_{S}}{1-Z_{S}}=\frac{\tilde{Z}-\hat{Z}_{S}}{1-\hat{Z}_{5}}, \\
T-1 & =\left(T_{0}-1\right) \xi+\frac{q}{S}(1-\xi)
\end{aligned}
$$

whereas for $\tilde{Z} \leq \tilde{Z}_{S}$

$$
\begin{aligned}
\hat{Y}_{\mathrm{F}} & =0 \text { and } \hat{Y}_{O}=1-\frac{Z}{Z_{S}}=1-\frac{\tilde{Z}}{\tilde{Z}_{S}}, \\
T-1 & =\left(T_{0}-1\right) \xi+\frac{q}{S}\left(\frac{\tilde{Z}}{\tilde{Z}_{S}}-\xi\right) .
\end{aligned}
$$

Equations (31) and (32) provide piece-wise linear relations for the evaluation of $\hat{Y}_{F}, \hat{Y}_{O}$, and $Z$ in terms of $\tilde{Z}$. In addition, the last relation in the equations gives $T$ in terms of $\tilde{Z}$ and $\xi$. When these are used together with (20) and (23), the flame location $\eta_{f}$ is determined as the value of $\eta$ where $\vec{Z}=\hat{Z}_{5}$, and $Z=Z_{5}$, while the flame temperature

$T_{f}-1=\left(T_{0}-1\right) \xi_{f}+\frac{q}{S}\left(1-\xi_{f}\right)$ 

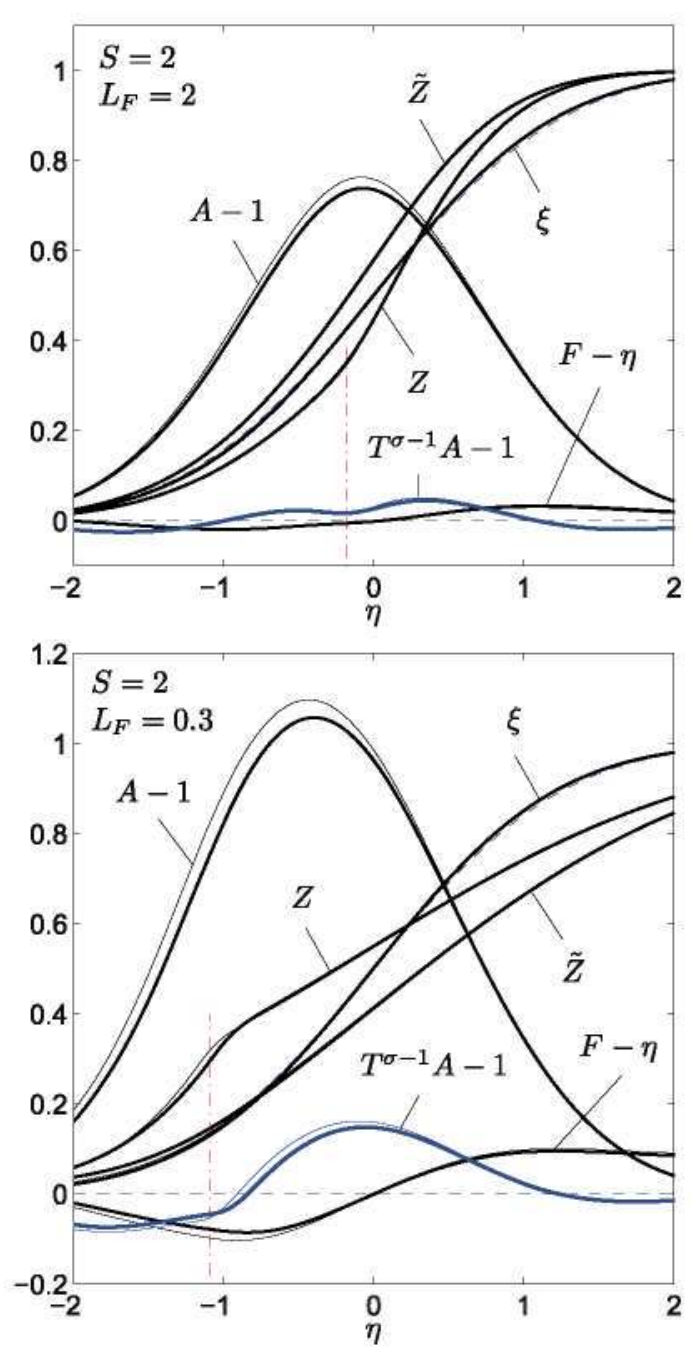

Fig. 2. The variation of the transverse mass flux $F$, strain rate $A$, excess enthalpy $\xi$, mixture fraction $Z$, and diffusion-weighted mixture fraction $\tilde{Z}$ as obtained from integrations of (13)-(17) (solid curves) for the parametric values of Fig. 1. The dashed lines represent the excess enthalpy profile $\xi=\frac{1}{2}[1+\operatorname{erf}(\eta / \sqrt{2})]$ obtained from (21) with the approximation $F=\eta$. The vertical dash-dotted lines indicate the flame location $\eta_{f}$ in the B-S limit.

is given by the value $\xi_{f}$ of the excess enthalpy at the flame, as follows from (20). Notice that the temperature and mass fractions, and also $Z$, given by (31) and (32) are continuous at the flame sheet, while their gradients have jumps there associated with the Dirac-delta reaction terms of their original conservation equations, as seen in the sample calculations of Figs. 1 and 2. However, the gradients of the conserved scalars $\xi$ and $\tilde{Z}$, determined by the sourceless Eqs. (21) and (25), are continuous at the flame sheet, as shown by (28) and (30). Their values at the flame sheet, $\widetilde{Z}=\widetilde{Z}_{S}$, can be used to evaluate the fuel and oxygen consumption rates per unit flame surface

$m_{\mathrm{F}}=\frac{m_{\mathrm{O}}}{\mathrm{S}}=\left.\frac{1}{L_{\mathrm{F}}} \frac{\mathrm{d} \hat{Y}_{\mathrm{F}}}{\mathrm{d} \eta}\right|_{\eta_{f}^{+}}=-\left.\frac{1}{\mathrm{~S}} \frac{\mathrm{d} \hat{Y}_{\mathrm{O}}}{\mathrm{d} \eta}\right|_{\eta_{f^{-}}}=\left.\frac{1+S / L_{\mathrm{F}}}{\mathrm{S}} \frac{\mathrm{d} \tilde{Z}}{\mathrm{~d} \eta}\right|_{\eta_{f}}$

and the fuel-side temperature gradient

$\left.\frac{\mathrm{d} T}{\mathrm{~d} \eta}\right|_{\eta_{f}^{+}}=\left.\left(T_{0}-1-q / S\right) \frac{\mathrm{d} \xi}{\mathrm{d} \eta}\right|_{\eta_{f}}$.

The latter expression can be combined with (30) to yield

$\gamma_{\mathrm{F}}=1-\gamma_{\mathrm{O}}=-\left.\frac{1}{q m_{\mathrm{F}}} \frac{\mathrm{d} T}{\mathrm{~d} \eta}\right|_{\eta_{f}^{+}}=\left.\frac{1}{m_{0}}\left(1-\frac{T_{0}-1}{q / S}\right) \frac{\mathrm{d} \xi}{\mathrm{d} \eta}\right|_{\eta_{f}}$ for the fractional amount of chemical heat release that is conducted towards the fuel side of the flame; while $\gamma_{0}$, close to unity when $S \gg 1$, represents the fraction transported to the air side.

The problem simplifies when $L_{\mathrm{F}}=1$, in which case the two scalars $Z$ and $\tilde{Z}$ are equal and satisfy the same Eq. (21) and boundary conditions as the conserved scalar $\xi$. The solution reduces to the integration of (13) and (14) together with an Eq. (21) for the passive scalar $\xi=Z=\tilde{Z}$. In particular, the peak temperature evaluated from (33) with $\xi_{f}=Z_{S}$ is

$T_{f}=T_{S}=1+\frac{q}{S+1}+\frac{\left(T_{0}-1\right)}{S+1}$,

where $T_{S}$ is the adiabatic flame temperature resulting from burning, at constant pressure, the mixture formed by combining in stoichiometric proportions the unit mass of the fuel stream with the mass of the air stream, at their corresponding initial temperatures. As can be seen in the sample B-S computations with $L_{\mathrm{F}} \neq 1$ shown in Fig. 1, the peak temperature $T_{f}$ differs from $T_{5}$.

\subsection{Burke-Schumann description for non-unity fuel Lewis numbers with the approximation $F=\eta$}

The use of the approximation $F=\eta$ for the velocity field in (21) and (25) allows us to derive analytic predictions for $\eta_{f}, T_{f}, m_{\mathrm{F}}$, and $\gamma_{0}$, which are identical to those obtained with the assumption of constant density and constant transport properties in the supplemental online material of [7]. With $F=\eta$ the excess enthalpy obtained from integration of (21) reduces to

$\xi=\frac{T-1+(q / S)\left(\hat{Y}_{0}-1\right)}{T_{0}-1-q / S}=\frac{1}{2}[1+\operatorname{erf}(\eta / \sqrt{2})]$

whereas integration of (25) supplemented with (31) and (32) provides

$\hat{Y}_{\mathrm{F}}=0$ and $\hat{Y}_{\mathrm{O}}=1-\frac{Z}{Z_{S}}=1-\frac{\tilde{Z}}{\tilde{Z}_{S}}=1-\frac{1+\operatorname{erf}(\eta / \sqrt{2})}{1+\operatorname{erf}\left(\eta_{f} / \sqrt{2}\right)}$

$$
\text { for } \eta<\eta_{f}
$$

and

$\hat{Y}_{\mathrm{O}}=0$ and $\hat{Y}_{\mathrm{F}}=\frac{Z-Z_{S}}{1-Z_{S}}=\frac{\tilde{Z}-\tilde{Z}_{S}}{1-\tilde{Z}_{S}}=1-\frac{1-\operatorname{erf}\left(\eta \sqrt{L_{\mathrm{F}} / 2}\right)}{1-\operatorname{erf}\left(\eta_{f} \sqrt{L_{\mathrm{F}} / 2}\right)}$

for $\eta>\eta_{f}$

for the composition. Using (39) and (40) in (34) yields

$m_{\mathrm{F}}=\frac{m_{\mathrm{O}}}{S}=\frac{\sqrt{2 /\left(\pi L_{\mathrm{F}}\right)} \exp \left(-L_{\mathrm{F}} \eta_{f}^{2} / 2\right)}{1-\operatorname{erf}\left(\eta_{f} \sqrt{L_{\mathrm{F}} / 2}\right)}=\frac{(\sqrt{2 / \pi} / S) \exp \left(-\eta_{f}^{2} / 2\right)}{1+\operatorname{erf}\left(\eta_{f} / \sqrt{2}\right)}$

for the rates of reactant consumption. The last equation in (41) determines implicitly the flame location $\eta_{f}$, which can be used in (38) to yield

$\xi_{f}=\frac{1+\operatorname{erf}\left(\eta_{f} / \sqrt{2}\right)}{2}=\frac{T_{f}-(1+q / S)}{T_{0}-(1+q / S)}$

for $\xi_{f}$ and $T_{f}$ and in (36) to yield

$\gamma_{\mathrm{F}}=1-\gamma_{0}=\left(1-\frac{T_{0}-1}{q / S}\right) \frac{1+\operatorname{erf}\left(\eta_{f} / \sqrt{2}\right)}{2}=\left(1-\frac{T_{0}-1}{q / S}\right) \xi_{f}$

for $\gamma_{\mathrm{F}}$ and $\gamma_{\mathrm{O}}$, thereby completing the determination of the flame parameters. 
3.4. Variation of flame properties with the fuel diffusivity and fuel-feed dilution

The exponent $\sigma$ for the temperature dependence of the transport properties enters in the continuity and momentum Eqs. (13) and (14)-the latter involving also the Prandtl number Prwhich determine the transverse velocity $F$, Besides these transport parameters, the formulation in the limit $B / A_{A} \rightarrow \infty$ involves as main parameters $S, q, L_{\mathrm{F}}$, and the fuel-to-air temperature ratio $T_{0}$. The numerical integrations, for the realistic values $\operatorname{Pr}=0.7$ and $\sigma=0.7$, are restricted here to the case of equal feed temperature $\left(T_{0}=1\right)$. In selecting the other parameters the reader should note that the values of $S$ and $q$ vary linearly with $Y_{\mathrm{F}_{0}}$, so that their ratio $q / S=q^{\prime} Y_{\mathrm{O}_{2} \mathrm{~A}} /\left(c_{p} T_{\mathrm{A}}^{\prime} s\right)$ is independent of the degree of dilution of the fuel stream. In fuel-air systems the resulting value is very similar for different fuels, including hydrocarbons and hydrogen, for which $q^{\prime} / s \simeq 13-15 \mathrm{MJ} / \mathrm{kg}$ yielding $q / S \simeq 10$ when $Y_{\mathrm{O}_{2} \mathrm{~A}}=0.232$, $c_{p}=1200 \mathrm{~J} /(\mathrm{kg} \mathrm{K})$, and $T_{\mathrm{A}}^{\prime}=300 \mathrm{~K}$ are used in the evaluations. This invariance is used in selecting the parameters in the computations below, so that for each value of $S$ the nondimensional heat-release parameter $q$ is given by $q=10 S$, representative of all fuel-air diffusion flames considered here. When this is implemented, only the two parameters $L_{\mathrm{F}}$ and $S$, respectively measuring the diffusivity of the fuel and the dilution of the fuel feed, need to be considered in evaluating the main flame properties in the limit of infinitely fast reaction,

The values of $\eta_{f}, T_{f}, m_{\mathrm{F}}$, and $\gamma_{\mathrm{O}}$ obtained numerically by integration of (13), (14), (21) and (25) supplemented with (31) and (32) are shown in Fig. 3. The figure allows us to evaluate the dependences of the different flame properties on the fuel Lewis number and on the fuel-feed dilution. To facilitate the representation, the overall stoichiometric ratio $S$ is replaced in the plots by the stoichiometric value of the modified mixture fraction $\tilde{Z}_{S}=$ $1 /\left(1+S / L_{\mathrm{F}}\right)$. The range of values of the fuel Lewis number considered $0.3 \leq L_{\mathrm{F}} \leq 2.5$ covers adequately the conditions encountered in typical combustion applications. Besides results of numerical integrations, indicated by the solid curves, the plots include as dashed curves the approximate analytic predictions obtained from (41)-(43). The degree of agreement displayed in the plots is remarkable, thereby further underscoring the utility of the weighted coordinate $\eta$ in analyzing counterflow flames.

The plots reveal the significant influence of the differentialdiffusion effects on flame temperatures. While the temperature increment $T_{f}-1$ with $L_{\mathrm{F}}=1$ remains always equal to the adiabatic value $q /(S+1)$, the peak temperatures are subadiabatic, i.e. $T_{f}-$ $1<q /(S+1)$, for $L_{\mathrm{F}}>1$ and superadiabatic, i.e. $T_{f}-1>q /(S+1)$, for $L_{\mathrm{F}}<1$. These observations are consistent with the classical reasoning according to which for $L_{\mathrm{F}}>1$ the rate at which the fuel diffuses into the reaction layer is smaller than the rate at which the heat is removed, thereby resulting in a temperature decrease, while the opposite behavior is found for $L_{\mathrm{F}}<1$. It is also of interest that this effect is more pronounced for moderately dilute flames that sit close to the stagnation plane, as can be seen by observing the accompanying plots of flame location $\eta_{f}$. It is worth pointing out that caution should be exerted when extrapolating the quantitative results presented here, in that the values given are specific to the counterflow and cannot be applied directly to other configurations, where $T_{f}$ varies along the flame surface.

\section{Extinction of diffusion flames in the diffusion-flame regime}

\subsection{Finite-rate effects for large activation energy}

Finite-rate effects in the diffusion-flame regime are negligible for large values of the Damköhler number, defined tentatively by multiplying the strain time $A_{\mathrm{A}}^{-1}$ by the characteristic reaction rate $B \exp \left(-T_{a} / T_{f}\right)$, evaluated with the $\mathrm{B}-\mathrm{S}$ flame temperature $T_{f}$. For large values of this tentative Damköhler number, $\left(B / A_{A}\right) \exp \left(-T_{a} / T_{f}\right)$, the reaction layer is very thin, with small mass fractions and small temperature differences from $T_{f}$, which grow to values of the order of the Frank-Kamenetskii temperature, $T_{f}^{2} / T_{a}$, when approaching the extinction conditions.

For large values of the activation energy, or values of the activation temperature $T_{a}$ large compared with the flame temperature $T_{f}$, flame extinction in the diffusion-flame regime [5] occurs with small departures from the equilibrium solution described above. Extinction is associated with small decrements of the temperature in the reaction layer from the peak value $T_{f}$, which when of order $T_{f}^{2} / T_{a}$ are sufficient to reduce the reaction rate by a factor $e$, as can be inferred from the exponential temperature dependence in (2). The ratio of the characteristic temperature increase by the chemical reaction, given by $q /(S+1)$, to this Frank-Kamenetskii temperature, $T_{f}^{2} / T_{a}$, defines the Zel'dovich number

$\beta=\frac{T_{a}}{T_{f}^{2}} \frac{q}{S+1}$,

of order $T_{a} / T_{f}$ for large $S$, as the relevant large parameter in the asymptotic description. The chemical reaction is confined to a thin layer of relative thickness $\beta^{-1}$, where we find small reactant mass fractions of order $\hat{Y}_{\mathrm{F}} \sim \beta^{-1}$ and $\hat{Y}_{\mathrm{O}} \sim \beta^{-1}$. These estimates, together with the requirement that the inner structure of the reaction layer is given by a balance between reaction and diffusion, can be used to provide from (17) the order of magnitude of the extinction strain rate

$A_{\mathrm{A}} \sim T_{f}^{\sigma-1}(S+1) \beta^{-3} B \exp \left(-T_{a} / T_{f}\right)$.

This estimate shows how the asymptotic limit of large activation energies, $T_{a} / T_{f} \gg 1$, requires simultaneous asymptotically large values of the frequency factor $B$, resulting in the balance (45) to ensure that the temperature decrements are of order $T_{f}^{2} / T_{a}=$ $\beta^{-1} q /(S+1)$ in the reaction layer.

The asymptotic description for $\beta \gg 1$ implies expansions in powers of $\beta^{-1}$ for the different variables in the inner reaction layer and in the outer convective-diffusive layers. The determination of the critical strain rate at extinction, if we are ready to accept errors of order $1 / \beta$, requires consideration of only two terms in the expansions of the dependent variables in the inner and outer layers. These two-term expansions begin, at leading order, with the B-S description; although the outer perturbations correspond to chemically frozen flow because of the associated low temperature.

\subsection{The inner layer}

The leading-order description of the thin reaction layer begins with the zero values of the mass fractions $\hat{Y}_{\mathrm{F}}$ and $\hat{Y}_{\mathrm{O}}$ and the flamesheet temperature $T_{f}$, corresponding to the B-S limit, but small perturbations of order $1 / \beta$ in the reaction layer have to be taken into account for their description, with relative errors of order $1 / \beta$ in the extinction regime, using the reaction-diffusion balance and leaving out the convective term, which is smaller by a factor $1 / \beta$. In the inner reaction layer, the first perturbations to the leadingorder $\mathrm{B}-\mathrm{S}$ flame are rescaled to give normalized variables of order unity according to $\zeta=\beta\left(\eta-\eta_{f}\right), y_{\mathrm{F}}=\beta \hat{\mathrm{Y}}_{\mathrm{F}}, y_{\mathrm{O}}=\beta \hat{\mathrm{Y}}_{\mathrm{O}}$, and $\theta=$ $T_{a}\left(T-T_{f}\right) / T_{f}^{2}=\beta\left(T-T_{f}\right) /[q /(S+1)]$. At the order pursued here, the determination of the extinction conditions involves only the terms in the above expansions, which are determined by integration of the corresponding reaction-diffusion equations

$-\frac{S}{S+1} \frac{\mathrm{d}^{2} \theta}{\mathrm{d} \zeta^{2}}=\frac{\mathrm{d}^{2} y_{\mathrm{O}}}{\mathrm{d} \zeta^{2}}=\frac{S}{L_{\mathrm{F}}} \frac{\mathrm{d}^{2} y_{\mathrm{F}}}{\mathrm{d} \zeta^{2}}=\frac{S \beta^{-3} B}{T_{f}^{1-\sigma} A_{S}} e^{-T_{a} / T_{f}} y_{\mathrm{F}} y_{\mathrm{O}} e^{\theta}$ 

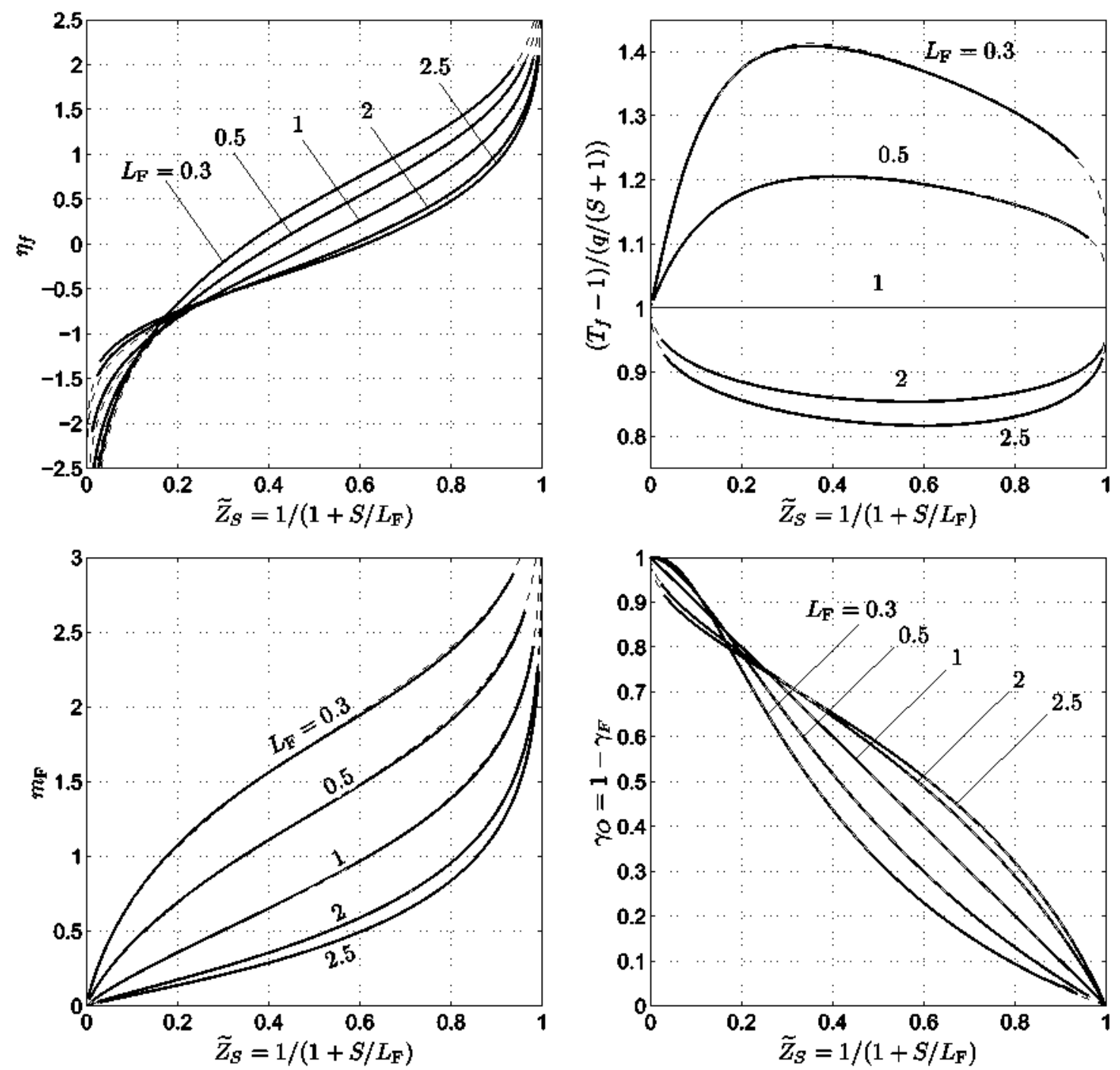

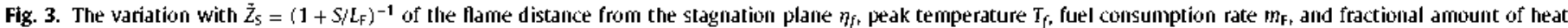

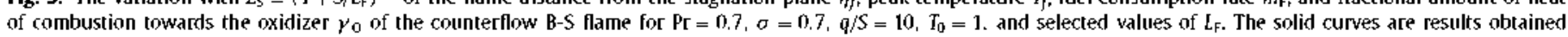

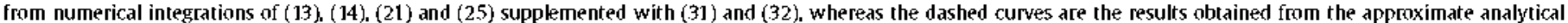
formulas (41)-(43)

obtained from (15)-(17); notice that the Arrhenius exponent is simplified to its linearized form $\theta$. The boundary conditions for integration of (46), given by

$$
\begin{aligned}
& \frac{\mathrm{d} \theta}{\mathrm{d} \zeta}=-\left(1-\gamma_{0}\right)(S+1) m_{\mathrm{F}}, \quad \frac{1}{L_{\mathrm{F}}} \frac{\mathrm{d} y_{\mathrm{F}}}{\mathrm{d} \zeta}=m_{\mathrm{F}}, \frac{\mathrm{d} y_{0}}{\mathrm{~d} \zeta}=0 \\
& \text { as } \zeta \rightarrow \infty \\
& \frac{\mathrm{d} \theta}{\mathrm{d} \zeta}=\gamma_{0}(S+1) m_{\mathrm{F}}, \quad \frac{\mathrm{d} y_{\mathrm{F}}}{\mathrm{d} \zeta}=0, \frac{\mathrm{d} y_{0}}{\mathrm{~d} \zeta}=-S m_{\mathrm{F}} \\
& \quad \text { as } \zeta \rightarrow-\infty
\end{aligned}
$$

follow from matching with the gradients of the B-S solution in the outer regions. In the intermediate matching region-for large $|\zeta|$ and small $\left|\eta-\eta_{j}\right|$-the inner variables take the form

$$
\begin{aligned}
& \theta=-\left(1-\gamma_{0}\right)(S+1) m_{\mathrm{F}} \zeta+\theta^{+}, \quad y_{\mathrm{F}}=m_{\mathrm{F}} L_{\mathrm{F}} \zeta+y_{\mathrm{F}}^{+}, \\
& y_{0}=y_{0}^{+} \text {as } \zeta \rightarrow \infty \\
& \theta=\gamma_{0}(S+1) m_{\mathrm{F}} \zeta+\theta^{-}, \quad y_{\mathrm{F}}=y_{\mathrm{F}}^{-}, y_{0}=-S m_{\mathrm{F}} \zeta+y_{0}^{-} \\
& \text {as } \zeta \rightarrow-\infty
\end{aligned}
$$

corresponding to (47). These expressions involve six unknown constants, namely, the reactant leakages $y_{0}^{+}$and $y_{\mathrm{F}}^{-}$and the apparent shifts $y_{\mathrm{F}}^{+}, y_{0}^{-}, \theta^{+}$, and $\theta^{-}$.

When the first two equations in (46) are integrated once, using the boundary conditions given in (47), the result is

$\frac{S+1}{S} \frac{d y_{0}}{d \zeta}+\frac{d \theta}{d \zeta}=-\gamma_{F}(S+1) m_{F}$ and $\frac{S}{L_{F}} \frac{d y_{F}}{d \zeta}-\frac{d y_{0}}{d \zeta}=S m_{F}$

while a second quadrature provides the relationships

$\frac{S+1}{S} y_{0}+\theta=-\gamma_{\mathrm{F}}(S+1) \mathrm{m}_{\mathrm{F}} \zeta+C_{1}$ and $\frac{S}{L_{\mathrm{F}}} y_{\mathrm{F}}-y_{0}=S m_{\mathrm{F}} \zeta+C_{2}$,

valid everywhere across the reaction layer. The two integration constants $C_{1}$ and $C_{2}$ are related to those in (48) by

$C_{1}=\frac{S+1}{S} y_{0}^{+}+\theta^{+}=\frac{S+1}{S} y_{0}^{-}+\theta^{-}$and

$C_{2}=\frac{S}{L_{\mathrm{F}}} y_{\mathrm{F}}^{+}-y_{0}^{+}=\frac{S}{L_{\mathrm{F}}} y_{\mathrm{F}}^{-}-y_{0}^{-}$. 
obtained by substitution of (48) into (50). The interpretation of these constants becomes more evident when the two relations (50) are written in terms of the excess enthalpy and diffusionweighted mixture fraction to give the expressions

$\xi-\xi_{f}=\left.\frac{\mathrm{d} \xi}{\mathrm{d} \eta}\right|_{\eta_{f}}\left(\eta-\eta_{f}\right)+\beta^{-1} \frac{S}{(S+1)} \frac{C_{1}}{\left[1-\left(T_{0}-1\right) /(q / S)\right]}$

and

$\tilde{Z}-\tilde{Z}_{S}=\left.\frac{\mathrm{d} \tilde{Z}}{\mathrm{~d} \eta}\right|_{\eta_{f}}\left(\eta-\eta_{f}\right)+\beta^{-1} \tilde{Z}_{S} C_{2}$,

which indicate that the terms of order $\beta^{-1}$ measured by $C_{1}$ and $C_{2}$, to be calculated as part of the solution, correspond to apparent shifts in the levels of $\xi$ and $\tilde{Z}$ at the flame from their B-S values $\xi_{f}$ and $\tilde{Z}_{S}$, associated with the leakage of the reactants through the reaction layer. As can be seen in (21), the perturbations to $\xi$ in the presence of finite-rate effects are only due to the modifications of the transverse velocity $F$, associated with the small perturbations in its source term. The resulting perturbations are neglected when the approximation $F=\eta$ is used in the computation of $\xi$ to yield $\xi=[1+\operatorname{erf}(\eta / \sqrt{2})] / 2$, in which case $C_{1}=0$, as discussed below in Section 4.5 .

\subsection{The canonical problem}

Using (50) to write the reaction rate in terms of $y_{\mathrm{p}}$ reduces the last equation in (46) to

$$
\begin{aligned}
\frac{\mathrm{d}^{2} y_{\mathrm{F}}}{\mathrm{d} \zeta^{2}}= & \frac{\beta^{-3} L_{\mathrm{F}}}{T_{f}^{1-\sigma}} \frac{B}{A_{\mathrm{A}}} e^{-E / R T_{f}^{\prime}} \exp \left(C_{1}+\frac{S+1}{S} C_{2}\right) y_{\mathrm{F}}\left(\frac{S}{L_{\mathrm{F}}} y_{\mathrm{F}}-S m_{\mathrm{F}} \zeta-C_{2}\right) \\
& \times \exp \left[-\frac{S+1}{L_{\mathrm{F}}} y_{\mathrm{F}}+\gamma_{\mathrm{O}}(S+1) m_{\mathrm{F}} \zeta\right]
\end{aligned}
$$

Introducing the normalized coordinate $\varsigma$ and the rescaled fuel variable $y$, defined by

$\varsigma=(S+1) m_{\mathrm{F}} \zeta+\frac{S+1}{S} C_{2}$ and $y=y_{\mathrm{F}} \frac{S+1}{L_{\mathrm{F}}}$,

so that

$\theta=-y+\gamma_{O} \varsigma+C_{1}+\frac{S+1}{S} \gamma_{\mathrm{F}} C_{2}$ and $y_{\mathrm{O}}=\frac{S}{S+1}(y-\varsigma)$,

enables the problem to be written in the canonical form

$\frac{\mathrm{d}^{2} y}{\mathrm{~d} \varsigma^{2}}=\Lambda y(y-\varsigma) e^{-y+\gamma_{0} \varsigma}\left\{\begin{array}{lll}y-\varsigma=y^{+} & \text {as } & \zeta \rightarrow+\infty \\ y=y^{-} & \text {as } & \zeta \rightarrow-\infty\end{array}\right.$

in terms of the reactant leakages

$y^{+}=y_{\mathrm{O}}^{+}(S+1) / S$ and $y^{-}=y_{\mathrm{F}}^{-}(S+1) / L_{\mathrm{F}}$.

The balance between chemical reaction and diffusion is measured in (56) by a canonical Damköhler number

$\Lambda=\mathcal{D} \exp \left(C_{1}+\frac{S+1}{S} \gamma_{\mathrm{F}} C_{2}\right)$

involving $C_{1}$ and $C_{2}$, unknown constants appearing in the first perturbations in the matching layer with the outer chemically frozen regions. The parameter $\Lambda$ is written in (58) in terms of the relevant Damköhler number

$D=\frac{S \beta^{-3} L_{\mathrm{F}} T_{f}^{\sigma-1}}{(S+1)^{3} m_{\mathrm{F}}^{2}} \frac{B}{A_{\mathrm{A}}} e^{-T_{a} / T_{f}}=\frac{L_{\mathrm{F}} T_{f}^{\sigma-1}}{m_{0}^{2}}\left(\frac{T_{f}}{T_{a}}\right)^{3}\left(\frac{T_{f}}{q / S}\right)^{3} \frac{B}{A_{\mathrm{A}}} e^{-T_{a} / T_{f}}$, of order unity near extinction, a nondimensional measure of the strain time $A_{\mathrm{A}}^{-1}$ ensuring when $\mathcal{D}$ is of order unity the correct balance between $B$ and the nondimensional activation energyrepresented by $T_{a} / T_{f}$ or $\beta$-associated with the double asymptotic limit implied in the estimate (45). The Damköhler number $\mathcal{D}$ involves the parameters $T_{f}$ and $m_{\mathrm{p}}$-the former also entering through the value of $\beta$ as defined in (44)-that are determined by the B-S description for non-unity values of the fuel Lewis number $L_{\mathrm{F}}$. Notice the strong dependence of $\mathcal{D}$ with $T_{f}$, reflected in the exponential term and in the $T_{f}^{5+\sigma}$ dependence of the prefactor. The value of $\Lambda$ defined in (58), due to the exponential factor, is different from the relevant Damköhler number $\mathcal{D}$. The constant $C_{1}+\gamma_{F} C_{2}(S+1) / S$, which carries the influence of the perturbations in the outer regions on the extinction process, represents a displacement of the temperature profile in the inner reactive region, as shown in (55b).

For a given value of the relative heat loss to the oxidizer side $\gamma_{0}$, determined from the B-S solution, the integration of (56) provides the inner structure of the reaction layer for different values of $\Lambda$, including the reactant leakages. For the values $0<\gamma_{0}<1$ that apply to most conditions of practical interest, two solutions are found for $\Lambda$ above a critical value $\Lambda_{E}$ and no solution exists for $\Lambda<\Lambda_{E}$. To handle effectively the resulting multiplicity, and thus determine accurately the solution near the turning point, instead of solving (56) for a known value $\Lambda$, the problem should be posed as that of finding the solution for a given fuel leakage $y^{-}$. Increasing the value of $y^{-}$in successive integrations provides the evolution of $\Lambda$ and $y^{+}$shown in Fig. 4. Results are shown for $\gamma_{0} \geq 0.5$. Corresponding results for $\gamma_{0} \leq 0.5$ can be easily obtained from Fig. 4 by replacing $\gamma_{0}$ by $\gamma_{\mathrm{F}}=1-\gamma_{0}$ and exchanging $y^{-}$and $y^{+}$.

Since the Damköhler number $\mathcal{D}$ is defined in terms of the values of $T_{f}$ and $m_{\mathrm{F}}$, provided by the B-S solution, the critical value of the strain rate at extinction can be evaluated from the critical value of $\mathcal{D}$ corresponding to the turning point of the curve giving the fuel leakage $y_{\mathrm{F}}^{-}$as a function of $\mathcal{D}$. As explained below, when the Lewis number of the fuel is unity, the case considered in [5], the constant $C_{1}+\gamma_{\mathrm{P}} C_{2}(S+1) / S$ appearing in (58) is identically zero, in which case $\mathcal{D}=\Lambda$ and the critical strain rate can be obtained directly from the canonical solution given in Fig. 4. When $L_{\mathrm{F}} \neq 1$, however, the perturbations in the outer regions, associated with the leakage of reactants, introduce a nonnegligible correction to the critical extinction conditions through a nonzero value of $C_{1}+\gamma_{\mathrm{F}} C_{2}(S+1) / S$, and the determination of the extinction conditions requires consideration of the perturbations in the outer layers, a problem addressed in the following section.

\subsection{The outer chemically frozen regions}

When considering the outer solution, the reaction layer appears as a sheet located at $\eta_{f}$. Different expansions must be used for the different flow variables on each side of the flame sheet. In the following, the terms in the expansion on the fuel side of the mixing layer (i.e., for $\eta_{f} \leq \eta<+\infty$ ) will be denoted by the superscript + . while those on the air side (i.e., for $-\infty<\eta \leq \eta_{f}$ ) will be denoted by the superscript - , giving for instance

$$
\begin{array}{rlrl}
T= & T_{0}^{+}+\beta^{-1} T_{1}^{+}, \hat{Y}_{\mathrm{F}}=Y_{\mathrm{F} 0}^{+}+\beta^{-1} Y_{\mathrm{F} 1}^{+}, & \hat{Y}_{\mathrm{O}}=Y_{\mathrm{OO}}^{+}+\beta^{-1} Y_{\mathrm{O} 1}^{+} \\
& \left(\eta_{f} \leq \eta<+\infty\right) \\
T= & T_{0}^{-}+\beta^{-1} T_{1}^{-}, \hat{Y}_{\mathrm{F}}=Y_{\mathrm{F} 0}^{-}+\beta^{-1} Y_{\mathrm{F} 1}^{-}, & & \hat{Y}_{0}=Y_{\mathrm{O} 0}^{-}+\beta^{-1} Y_{01}^{-} \\
& \left(-\infty<\eta \leq \eta_{f}\right)
\end{array}
$$

for the first two terms in the expansions for the temperature and reactant mass fractions, with similar expansions introduced for the velocity field. The leading terms in the expansions correspond to the B-S solution described above, so that $Y_{\mathrm{F} 0}^{-}=0$ for $\eta<\eta_{f}$ and 

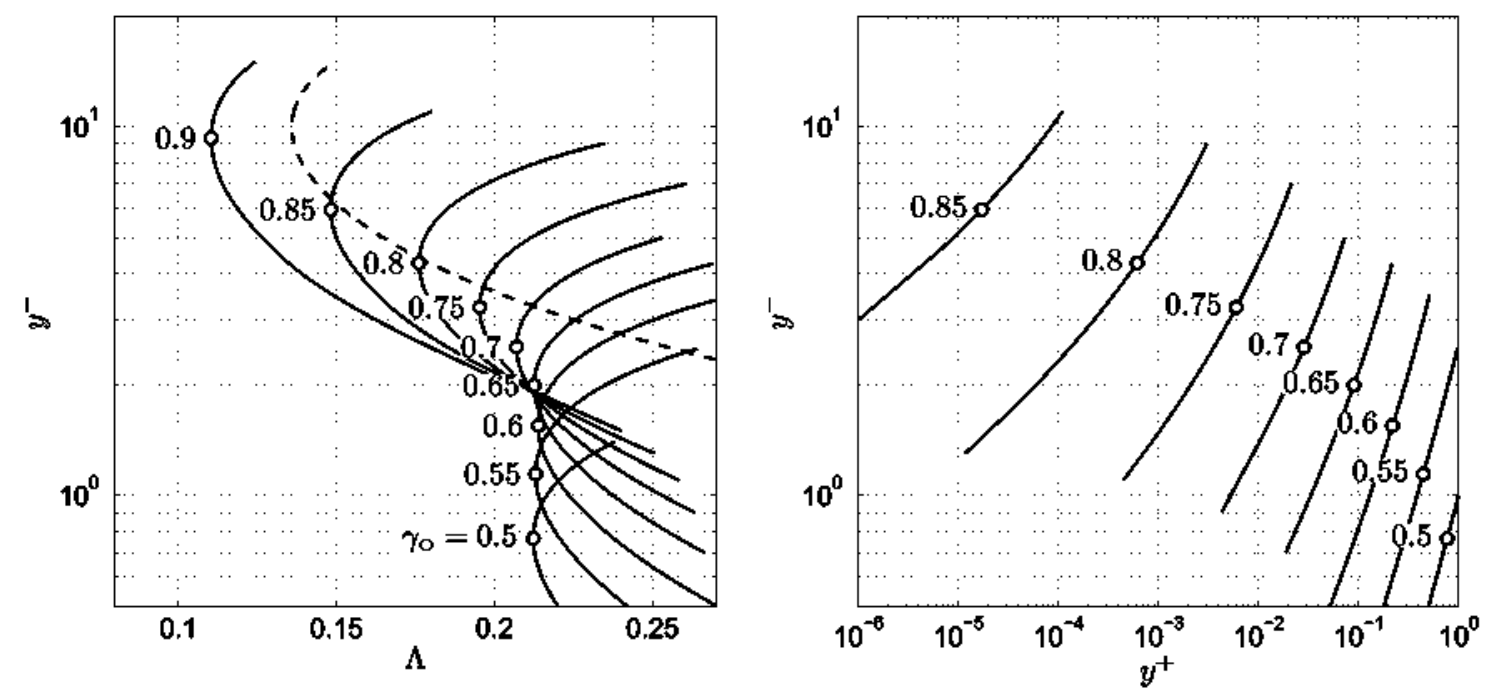

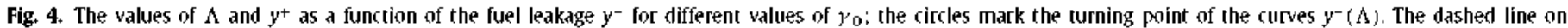
the left plot represents the curve $y^{-}(\Lambda)$ predicted by $(71)$ in the premixed-flame regime for $\gamma_{0}=1-\gamma_{\mathrm{F}}=0.9$.

$Y_{00}^{+}=0$ for $\eta>\eta_{f}$, as follows from (27). The resulting zerothorder solution, which corresponds to that represented with dashed curves in Fig. 1, determines in particular the reaction-sheet location $\eta_{f}$ along with the additional flame properties shown in Fig. 3.

The first-order corrections are due to finite-rate effects in the reaction layer, leading in particular to nonzero boundary values $Y_{\mathrm{F} 1}^{-}$and $Y_{01}^{+}$, for the functions $Y_{\mathrm{F} 1}^{-}$and $Y_{O 1}^{+}$at $\eta=\eta_{f}$, associated with reactant leakage of order $\beta^{-1}$ across the flame. The boundary values for the first-order perturbations in the outer regions $T_{1_{f}}^{ \pm}$, $Y_{\mathrm{F} 1 f}^{ \pm}$, and $Y_{\mathrm{O} \mathrm{I}_{f}}^{ \pm}$are related to the reactant leakages $y_{\mathrm{O}}^{+}$and $y_{\mathrm{F}}^{-}$and the apparent shifts $y_{\mathrm{F}}^{+}, \theta^{+}, y_{0}^{-}$, and $\theta^{-}$of the inner solution by

$\theta^{ \pm}=\frac{T_{1_{f}}^{ \pm}}{q /(S+1)}, \quad y_{\mathrm{F}}^{ \pm}=Y_{\mathrm{F} 1_{f}}^{ \pm}, \quad$ and $y_{0}^{ \pm}=Y_{O 1_{f}}^{ \pm}$

as required at this order to achieve the matching of (48) with the outer solution $(60)$.

The equations involved in the computation of the functions $Y_{\mathrm{F} 1}^{ \pm}$. $Y_{O 1}^{ \pm}$, and $T_{1}^{ \pm}$and of the associated velocity perturbations are obtained by linearizing (13)-(17) without the reaction terms. The boundary values and their gradients on both sides of the flame must satisfy a number of constraints associated with the conservation equations. For instance, a first integral of the chemistry-free equation (22) with use made of the expansions (60) provides

$$
\begin{aligned}
& F\left(\eta_{f}\right)\left[S\left(\mathrm{Y}_{\mathrm{F} 1_{f}}^{+}-\mathrm{Y}_{\mathrm{F} 1_{f}}^{-}\right)-\left(\mathrm{Y}_{\mathrm{O} 1_{f}}^{+}-\mathrm{Y}_{\mathrm{O} 1_{f}}^{-}\right)\right] \\
& +\frac{S}{\mathrm{LF}}\left(\left.\frac{\mathrm{d} \mathrm{F}_{\mathrm{F} 1}^{+}}{\mathrm{d} \eta}\right|_{\eta_{f}}-\left.\frac{\mathrm{dY}-\overline{\mathrm{Fl}}}{\mathrm{d} \eta}\right|_{\eta_{f}}\right)-\left(\left.\frac{\mathrm{d} \mathrm{Y}_{\mathrm{O} 1}^{+}}{\mathrm{d} \eta}\right|_{\eta_{f}}-\left.\frac{\mathrm{d} \mathrm{Y}_{01}^{-}}{\mathrm{d} \eta}\right|_{\eta_{f}}\right)=0 .
\end{aligned}
$$

where $F\left(n_{j}\right)$ is to be evaluated from the leading-order solution.

4.5. The perturbations in the outer regions with the approximation $F=\eta$

The solution to the first-order outer perturbations simplifies greatly when the approximation $F=\eta$ is adopted for the velocity field, leading to explicit expression for the constants $C_{1}$ and $C_{2}$. It can be noted that the simplified form of (21) when $F=\eta$ has the exact solution given in (38) valid for all $\eta$, so that in (52) $C_{1}=0$. The outer perturbations $T_{1}(\eta)$ and $Y_{01}(\eta)$ are related by $T_{1}^{ \pm}(\eta)=$ $-(q / S) Y_{01}^{ \pm}(\eta)$, and from $(61)$ it follows that $\theta^{ \pm}+(S+1) y_{0}^{ \pm} / S=0$.

Using the approximation $F=\eta$ in the transport equations for $Y_{\mathrm{F} 1}^{ \pm}$and $Y_{\mathrm{O} 1}^{ \pm}$, integrated with the boundary conditions $Y_{\mathrm{F} 1}^{ \pm}( \pm \infty)=$
$Y_{01}^{ \pm}( \pm \infty)=0$ and $Y_{\mathrm{F} 1}^{ \pm}\left(\eta_{f}\right)-Y_{\mathrm{F}_{1}}^{ \pm}=Y_{01}^{ \pm}\left(\eta_{f}\right)-Y_{01_{f}}^{ \pm}=0$ provides

$\frac{Y_{\mathrm{Fl}}^{+}}{\mathrm{Y}_{\mathrm{F} 1_{f}}^{+}}=\frac{1-\operatorname{erf}\left(\eta \sqrt{L_{\mathrm{F}} / 2}\right)}{1-\operatorname{erf}\left(\eta_{f} \sqrt{L_{\mathrm{F}} / 2}\right)}$ and $\frac{Y_{O 1}^{+}}{Y_{O 1_{f}}^{+}}=\frac{1-\operatorname{erf}(\eta / \sqrt{2})}{1-\operatorname{erf}\left(\eta_{f} / \sqrt{2}\right)}$

$$
\text { for } \eta>n_{f}
$$

and

$\frac{Y_{\mathrm{F} 1}^{-}}{Y_{\mathrm{F} 1_{f}}^{-}}=\frac{1+\operatorname{erf}\left(\eta_{\left.\sqrt{L_{\mathrm{F}} / 2}\right)}\right.}{1+\operatorname{erf}\left(\eta_{f} \sqrt{L_{\mathrm{F}} / 2}\right)}$ and $\frac{Y_{O 1}^{-}}{Y_{O 1_{f}}^{-}}=\frac{1+\operatorname{erf}(\eta / \sqrt{2})}{1+\operatorname{erf}\left(\eta_{f} / \sqrt{2}\right)}$

$$
\text { for } \eta>\eta_{f}
$$

with associated gradients at the flame given by

$$
\begin{aligned}
& \left.\frac{\mathrm{dY} \mathrm{F}^{ \pm}}{\mathrm{d} \eta}\right|_{\eta_{f}}=\mp Y_{\mathrm{F} 1_{f}}^{ \pm} \frac{\sqrt{2 L_{\mathrm{F}} / \pi} \exp \left(-L_{\mathrm{F}} \eta_{f}^{2} / 2\right)}{1 \mp \operatorname{erf}\left(\eta_{f} \sqrt{L_{\mathrm{F}} / 2}\right)} \text { and } \\
& \left.\frac{\mathrm{dY} \mathrm{O}_{\mathrm{O}}^{ \pm}}{\mathrm{d} \eta}\right|_{\eta_{f}}=\mp \mathrm{Y}_{O 1_{f}}^{ \pm} \frac{\sqrt{2 / \pi} \exp \left(-\eta_{f}^{2} / 2\right)}{1 \mp \operatorname{erf}\left(\eta_{f} / \sqrt{2}\right)} .
\end{aligned}
$$

The above expressions can be used in (62) to give

$$
\begin{aligned}
& \frac{S Y_{\mathrm{F} 1_{f}}^{+}}{L_{\mathrm{F}}}\left[L_{\mathrm{F}} \eta_{f}-\frac{\sqrt{2 L_{\mathrm{F}} / \pi} \exp \left(-L_{\mathrm{F}} \eta_{f}^{2} / 2\right)}{1-\operatorname{erf}\left(\eta_{f} \sqrt{L_{\mathrm{F}} / 2}\right)}\right] \\
& -\frac{S Y_{\mathrm{F} 1_{f}}^{-}}{L_{\mathrm{F}}}\left[L_{\mathrm{F}} \eta_{f}+\frac{\sqrt{2 L_{\mathrm{F}} / \pi} \exp \left(-L_{\mathrm{F}} \eta_{f}^{2} / 2\right)}{1+\operatorname{erf}\left(\eta_{f} \sqrt{L_{\mathrm{F}} / 2}\right)}\right] \\
& -Y_{\mathrm{O} 1_{f}}^{+}\left[\eta_{f}-\frac{\sqrt{2 / \pi} \exp \left(-\eta_{f}^{2} / 2\right)}{1-\operatorname{erf}\left(\eta_{f} / \sqrt{2}\right)}\right] \\
& +Y_{O 1_{f}}^{-}\left[\eta_{f}+\frac{\sqrt{2 / \pi} \exp \left(-\eta_{f}^{2} / 2\right)}{1+\operatorname{erf}\left(\eta_{f} / \sqrt{2}\right)}\right]=0 .
\end{aligned}
$$

Substituting into (66) the expressions

$$
\begin{aligned}
& \frac{S \mathrm{~F}_{f}^{+}}{L_{\mathrm{F}}}=C_{2}+\frac{S}{S+1} y^{+}, \frac{S \mathrm{Y}_{\mathrm{F}_{f}}^{-}}{L_{\mathrm{F}}}=\frac{S}{S+1} y^{-}, Y_{\mathrm{O} 1_{f}}^{+}=\frac{S}{S+1} y^{+} . \\
& Y_{\mathrm{O}_{f}}^{-}=\frac{S}{S+1} y^{-}-C_{2},
\end{aligned}
$$



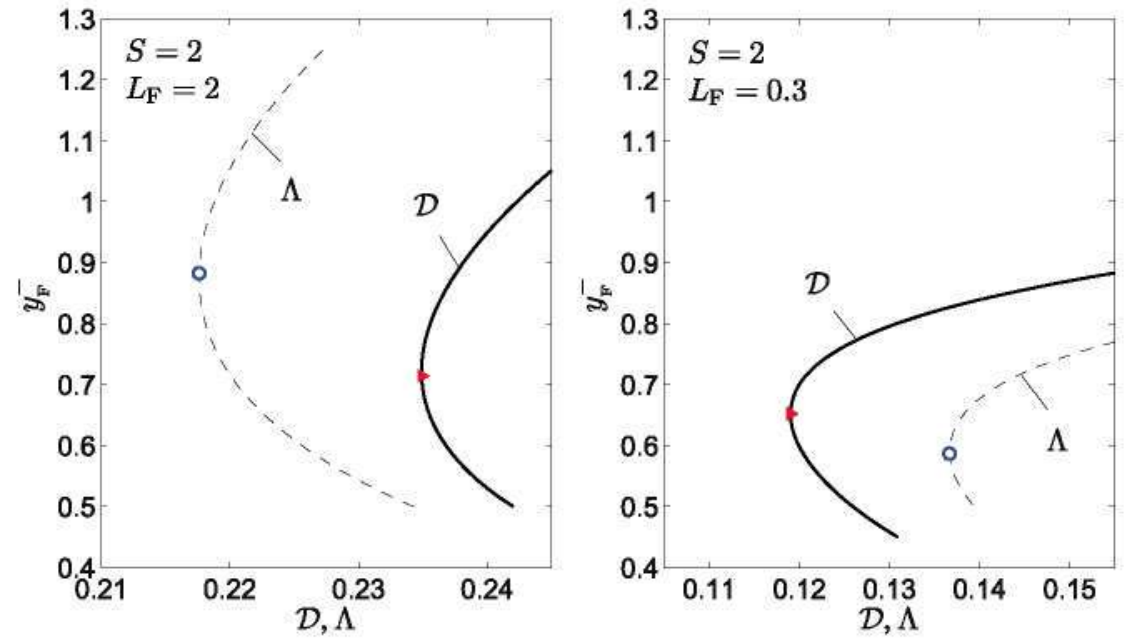

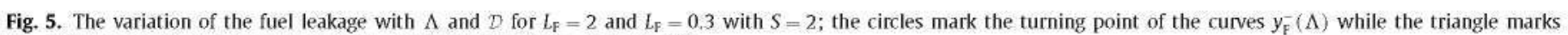
the extinction point, corresponding to the turning point of the curves $y_{\mathrm{F}}^{-}(\mathcal{D})$.

which follow from (51), (57), and (61), and solving for $C_{2}$ finally yields

$$
\begin{aligned}
\frac{S+1}{S} C_{2}= & {\left[\frac{\sqrt{2 / \pi} \exp \left(-\eta_{f}^{2} / 2\right)}{1+\operatorname{erf}\left(\eta_{f} / \sqrt{2}\right)}\right.} \\
& \left.+\frac{\sqrt{2 L_{\mathrm{F}} / \pi} \exp \left(-L_{\mathrm{F}} \eta_{f}^{2} / 2\right)}{1-\operatorname{erf}\left(\eta_{f} \sqrt{L_{\mathrm{F}} / 2}\right)}-\left(L_{\mathrm{F}}-1\right) \eta_{f}\right]^{-1} \\
& \times\left\{\left(y^{+}-y^{-}\right)\left(L_{\mathrm{F}}-1\right) \eta_{f}+\frac{\sqrt{2 / \pi} \exp \left(-\eta_{f}^{2} / 2\right)}{1-\operatorname{erf}^{2}\left(\eta_{f} / \sqrt{2}\right)}\right. \\
& \times\left[y^{+}+y^{-}+\left(y^{+}-y^{-}\right) \operatorname{erf}\left(\eta_{f} / \sqrt{2}\right)\right] \\
& -\frac{\sqrt{2 L_{\mathrm{F}} / \pi} \exp \left(-L_{\mathrm{F}} \eta_{f}^{2} / 2\right)}{1-\operatorname{erf}^{2}\left(\eta_{f} \sqrt{L_{\mathrm{F}} / 2}\right)} \\
& \left.\times\left[y^{+}+y^{-}+\left(y^{+}-y^{-}\right) \operatorname{erf}\left(\eta_{f} \sqrt{L_{\mathrm{F}} / 2}\right)\right]\right\},
\end{aligned}
$$

to be used in (58) with $C_{1}=0$ to relate the Damköhler numbers $\Lambda$ and $\mathcal{D}$.

\subsection{Evaluation of the extinction conditions}

For given values of $S, L_{\mathrm{F}}, q$, and $T_{0}$, the above results can be used to determine the critical value of the strain rate at extinction, given by the minimum value of $\mathcal{D}$ for which a solution exists, associated with an intermediate value of the fuel leakage $y_{F}^{-}$. The computation begins by considering the limit of infinitely fast reaction to determine the flame location $\eta_{f}$, the peak temperature $T_{f}$. the reactant consumption rates $m_{\mathrm{O}}$ and $m_{\mathrm{F}}=m_{\mathrm{O}} / S$, and the heatloss parameters $\gamma_{O}$ and $\gamma_{\mathrm{F}}=1-\gamma_{0}$. As shown above, these quantities can be evaluated with excellent accuracy by using the expressions (41)-(43) associated with the approximation $F=\eta$. The heat-loss parameter $\gamma_{0}$ can be used when solving the canonical problem (56), which gives the variation of $\Lambda$ and $y^{+}=y_{0}^{+}(S+1) / S$ with $y^{-}=y_{\mathrm{F}}^{-}(S+1) / L_{\mathrm{F}}$. The curve giving the variation of $\mathcal{D}$ with $y_{\mathrm{F}}^{-}$can then be obtained by using (58) together with $C_{1}=0$ and (68), giving the results shown in Figs. 5 and 6.

The effect of the outer perturbations on the critical extinction conditions for $L_{\mathrm{F}} \neq 1$ is illustrated in Fig. 5. The dashed curves represent the functions $y_{\mathrm{F}}^{-}(\Lambda)$ obtained from direct evaluation of the solution to the canonical problem (56) for the value of $\gamma_{0}$ corresponding to $S=2$ with $L_{\mathrm{F}}=2$ and $L_{\mathrm{F}}=0.3$, while the corrected extinction curves $y_{\mathrm{F}}^{-}(\mathcal{D})$ accounting for the outer perturbations are shown as solid curves. The differences between the resulting Damköhler numbers, associated with the nonzero values of $\mathrm{C}_{2}$, are seen to be moderately small for the two cases considered in the figure. It is also of interest that the turning point of the curve $\mathcal{D}\left(y_{\mathrm{F}}^{-}\right)$identifying extinction (i.e. the solid triangles on the solid curves) occurs at a value of $y_{F}^{-}$that differs from that of the turning point of the canonical curve $\Lambda\left(y_{\mathrm{F}}^{-}\right)$.

The variation of the extinction curves with dilution for $L_{\mathrm{F}}=2$ and $L_{\mathrm{F}}=0.3$ is shown in Fig. 6 . As can be seen, the corrections to the critical Damköhler number associated with the perturbations to the outer flow, induced by reactant leakage through the flame, are moderately small. Also of interest is that the value of the fuel leakage $y_{\mathrm{F}}^{-}$at extinction exhibits a non-monotonic dependence on $S$.

It is worth noting that, as shown in Fig. 4, for values of $S$ of order unity the values of the temperature gradients are not far from the symmetric case $\gamma_{0}=1-\gamma_{\mathrm{F}}=0.5$ and the extinction of the diffusion flame occurs with significant leakage of both reactants. The bifurcating unstable branch, for values of the Damköhler number large compared with the extinction value, has the structure of the partial-burning regime analyzed in [5]. For the Arrhenius overall exothermic reaction considered here the only possible solution for the decreasing temperature from its peak value has the same slope when the reaction becomes frozen on both sides of the reaction layer. It is of interest that this partial-burning regime was encountered recently by Norbert Peters and coworkers [16], and found to be stable, for the non-Arrhenius reaction associated with cool flames of alkanes, such as heptane, when the reaction rate is found to decrease with the peak temperature in a certain temperature range.

\subsection{Comparison with numerical results for finite values of $\beta$.}

The original set of Eqs. (13)-(18) was employed to compute the extinction curves for finite values of the activation energy. The integrations employed continuation methods to capture the turning point of the extinction curve [17]. To facilitate the comparisons with the results of the asymptotic analysis, the preexponential factor $B$ and the activation temperature $T_{a}$ were replaced in the numerical integrations by the Damköhler number $\mathcal{D}$ and the Zel'dovich number $\beta$ by writing the reaction-rate 

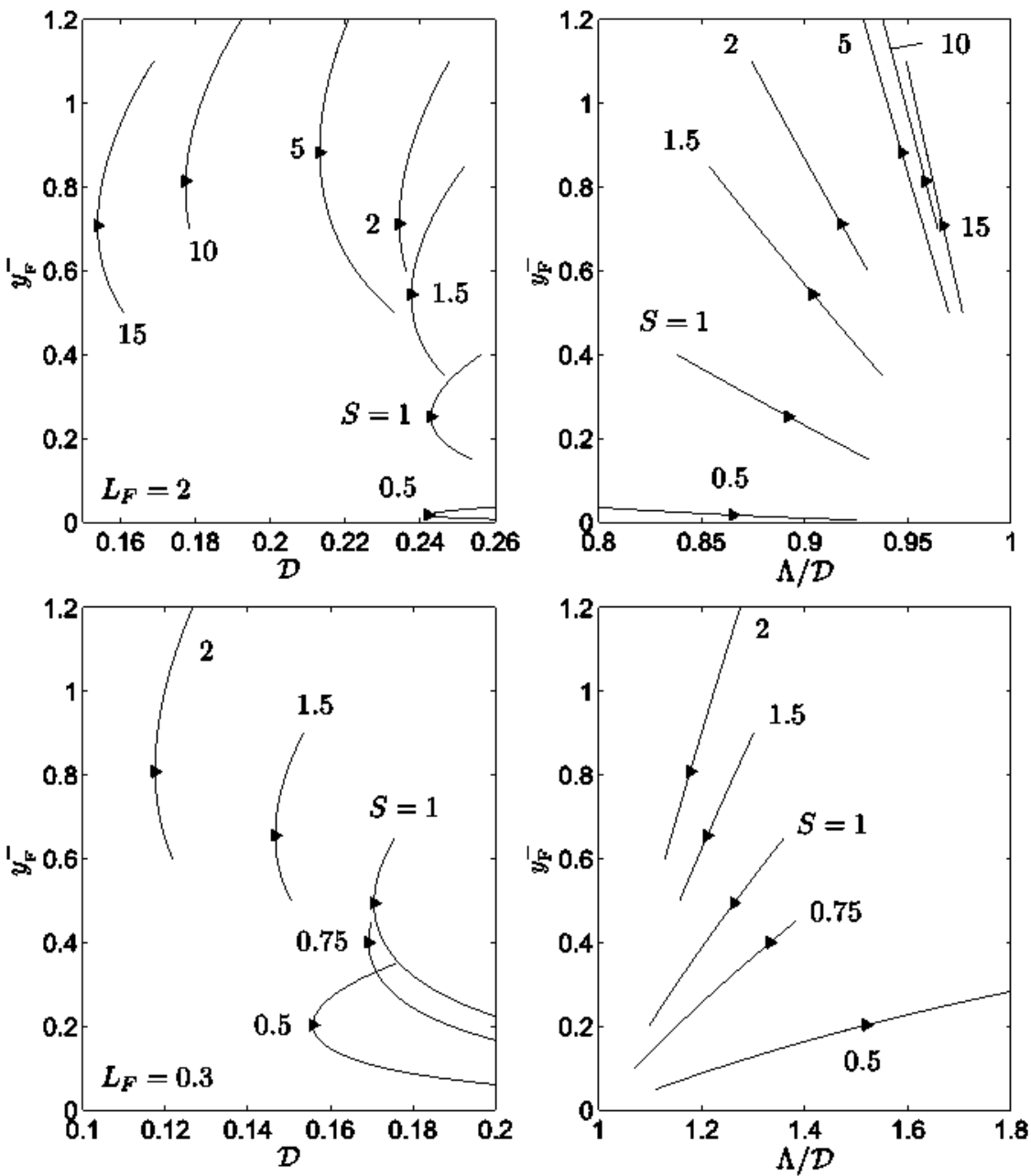

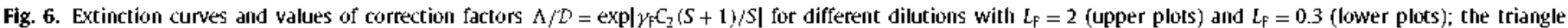
marks the extinction point on each curve.

constant in the form

$\frac{B}{A_{\mathrm{A}}} \mathrm{e}^{-T_{\mathrm{o}} / T}=\mathcal{T} \frac{(S+1)^{3} \mathrm{~m}_{\mathrm{F}}^{2}}{S L_{\mathrm{F}}} \mathrm{T}_{f}^{\sigma-1} \beta^{3} \exp \left(\beta \frac{T-T_{f}}{T} \frac{T_{f}}{q /(S+1)}\right)$.

For given values of $q, S$ and $L_{F}$, the $B-S$ solution was computed first to obtain the equilibrium values $T_{f}$ and $m_{\mathrm{F}}$. Then, the finitechemistry numerical simulations were carried out for a fixed value of the Zel'dovich number $\beta$ for different values of $D$ and the resulting curves of peak temperature versus $D$ were compared against the predictions of the asymptotic analysis corresponding to large $\beta$ in the distinguished extinction regime $T \sim 1$.

Figure 7 compares the value of $\theta_{\max }=\beta\left(T_{\max }-T_{f}\right) /[q /(S+1)]$ evaluated from the peak temperature $T_{\max }$ of the numerical integrations for several values of $\beta$ with the asymptotic prediction for large $\beta$ obtained from the local maximum of the curve $\theta(\zeta)$ evaluated from (55b) at extinction. The agreement of the asymptotic predictions with the numerical results is satisfactory in the two cases considered, with errors of order $\beta^{-1}$ that decrease for increasing $\beta$.

\section{Extinction of diffusion flames in the premixed-flame regime}

For large values of $S$ and small values of $L_{F}$ the B-S flame migrates towards the air side of the mixing layer; resulting in a small value, $\gamma_{\mathrm{F}} / \gamma_{0} \ll 1$, of the ratio of the temperature gradients to the fuel and the air side of the reaction layer, as seen in Fig. 3. Under these conditions, extinction of the diffusion flame occurs in a premixed-flame regime, because the drop in temperature from the $\mathrm{B}-\mathrm{S}$ value is not enough on the fuel side to allow the leakage of the oxygen through the thin reaction layer prior to flame extinction (i.e. note in Fig. 4 the negligibly small value of $y^{+}$at extinction when $1-\gamma_{0} \ll 1$ ). However, when approaching the extinction conditions by lowering the canonical Damköhler number $\Lambda$, the mass fraction of the leaking fuel has grown to large values of order $y^{-} \sim\left(1-\gamma_{0}\right)^{-1}$, thus lowering the flame peak temperature from the Burke-Schumann value $T_{f}$ by an amount of order $T_{f}^{2} / T_{q}$. The reaction layer is also displaced from the B-S position towards the fuel side, to a location given approximately by $\zeta=y^{-}$. This reaction layer separates a chemically frozen region with $y=y^{-}$from a region in chemical equilibrium with $y-\xi=y^{+}=0$.

Since for large $y^{-}$the fuel mass fraction $y$ changes only by a small amount, of order $1 / y^{-}$, across the reaction layer, one can evaluate the reaction rate with $y=y^{-}$when solving (56), and rewrite the problem in terms of the oxidizer mass fraction $\tilde{y}=$ $y-\zeta$ and the translated coordinate $\vec{\zeta}=\zeta-y^{-}$to give

$$
\frac{\mathrm{d}^{2} \tilde{y}}{\mathrm{~d} \tilde{\zeta}^{2}}=\Lambda y^{-} \exp \left(-\gamma_{\mathrm{F}} y^{-}\right) \tilde{y} e^{-\bar{y}} \quad\left\{\begin{array}{ll}
\tilde{y}=0 & \text { as } \quad \tilde{\xi} \rightarrow \infty \\
\tilde{y}+\tilde{\zeta} x=0 & \text { as } \quad \tilde{\xi} \rightarrow-\infty
\end{array},\right.
$$

involving $\gamma_{F}=1-\gamma_{0}$, the fraction of the heat released at the flame that is conducted towards the fuel side, a quantity that is small 

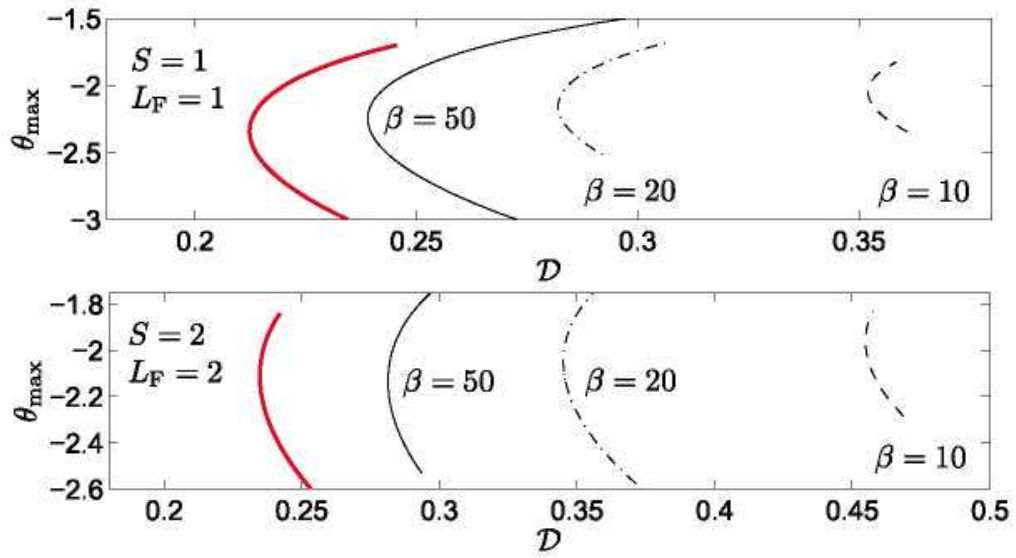

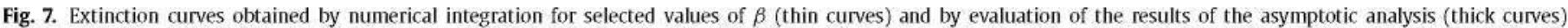
for $\left(L_{\mathrm{F}}, S\right)=(1,1)$ and $\left(L_{\mathrm{F}}, S\right)=(2,2)$ with $q / S=10$.

when $S \gg 1$. A first quadrature of this last equation yields

$\Lambda=\exp \left(\gamma_{\mathrm{F}} y^{-}\right) /\left(2 y^{-}\right)$,

a relation between the normalized fuel leakage $y^{-}$and $\Lambda$ valid for $\gamma_{\mathrm{F}} \ll 1$. This relation is plotted in Fig. 4 for $\gamma_{\mathrm{F}}=0.1$. Comparison with the curve $y^{-}(\Lambda)$ obtained from the numerical integration of the canonical problem (56) shows that the approximations leading to (71) result in an overprediction of the extinction Damköhler number by a factor of order $\gamma_{\mathrm{F}}$.

Since $y^{+}$is negligibly small, the constant $C_{2}$ giving $\mathcal{D}=$ $\Lambda \exp \left[-\gamma_{\mathrm{F}} C_{2}(S+1) / S\right]$ in terms of $\Lambda$, according to (58), leads to

$\frac{S+1}{S} C_{2}=\Gamma y^{-}$,

where

$\Gamma=\left[\frac{\sqrt{2 / \pi} \exp \left(-\eta_{f}^{2} / 2\right)}{1+\operatorname{erf}\left(\eta_{f} / \sqrt{2}\right)}-\frac{\sqrt{2 L_{\mathrm{F}} / \pi} \exp \left(-L_{\mathrm{F}} \eta_{f}^{2} / 2\right)}{1+\operatorname{erf}\left(\eta_{f} \sqrt{L_{\mathrm{F}} / 2}\right)}-\left(L_{\mathrm{F}}-1\right) \eta_{f}\right]$

$\times\left[\frac{\sqrt{2 / \pi} \exp \left(-\eta_{f}^{2} / 2\right)}{1+\operatorname{erf}\left(\eta_{f} / \sqrt{2}\right)}+\frac{\sqrt{2 L_{\mathrm{F}} / \pi} \exp \left(-L_{\mathrm{F}} \eta_{f}^{2} / 2\right)}{1-\operatorname{erf}\left(\eta_{f} \sqrt{L_{\mathrm{F}} / 2}\right)}-\left(L_{\mathrm{F}}-1\right) \eta_{f}\right]^{-1}$.

The factor $\Gamma$ turns out to be fairly small for undiluted fuel feed $(S \gg 1)$, as can be seen by evaluating $(73)$ for $-\eta_{f} \gg 1$.

Using (72) in (58) with $C_{1}=0$ yields

$D=\exp \left[\gamma_{\mathrm{F}}(1-\Gamma) y^{-}\right] /\left(2 y^{-}\right)$.

The turning point associated with extinction, corresponding to the minimum value of the curve $\mathcal{D}\left(y^{-}\right)$, can be obtained by differentiation of (74) to give

$\mathcal{D}_{e}=(\mathrm{e} / 2) \gamma_{\mathrm{F}}(1-\Gamma)$ and $y_{e}^{-}=1 /\left[\gamma_{\mathrm{F}}(1-\Gamma)\right]$

for the Damköhler number at extinction and its associated reduced fuel leakage, respectively. The first expression in (75) can be used together with the definition given in (59) and the B-S solution shown in Fig. 3 to determine the critical value of the strain rate at extinction $A_{\mathrm{A}}$ when extinction occurs in the premixed flame regime (i.e. for $\gamma_{\mathrm{F}} \ll 1$ ).

\section{Concluding remarks}

This paper gives a description of the differential-diffusion effects, associated with non-unity values of the Lewis number $L_{\mathrm{F}}$ of the fuel, on the structure of counterflow diffusion flames and their extinction when subject to strong strain. A heat-conductionweighted transverse coordinate $\eta$ is found to be a convenient choice for describing the steady counterflow problem, because when using $\eta$ the variations with temperature of the heat conductivity are eliminated from the convective and diffusive terms of the conservation equations for the temperature, mass fractions of the reactants, and for the strain rate $A$, given by the radial momentum equation. Although $T^{\sigma-1}$ appears as a factor in the reaction terms, it does not play a role in the B-S limit of fast rates, and it can be approximated by $T_{f}^{o-1}$ in the near-extinction regime.

When using $\eta$, the convective transport is associated with the mass flux $F$, whose variation with $\eta$ is given by the mass conservation equation (13), which has a source term, the strain rate $A$ multiplied by $T^{\sigma-1}$, due to the radial flux. It turns out that the difference of this source term from unity, shown in Fig. 2, is small, so that the approximation $F=\eta$ reduces the conservation equations to the form obtained when using the thermaldiffusive approximation of constant density and heat conductivity.

The overall irreversible reaction considered has a reactionrate constant, $B \exp \left(-T_{a} / T\right)$, involving a frequency factor $B$ and a strongly temperature-dependent Arrhenius exponential, because the activation temperature $T_{a}$ is moderately large when compared with the flame temperature $T_{f}$. The analysis begins with the description for infinitely large values of the ratio $\left(B / A_{\mathrm{A}}\right) \exp \left(-T_{a} / T_{f}\right)$ of the reaction rate to the flow strain rate $A_{\mathrm{A}}$. In this $\mathrm{B}-\mathrm{S}$ limit, the reaction layer appears as an infinitesimally thin flame sheet, which acts as a Dirac-delta concentrated source for the thermal energy, and a sink for the fuel and oxygen. The fuel-consumption rate $m_{\mathrm{F}}$ is determined by the convective and diffusive transport of the reactants to the flame sheet; giving a well-defined peak temperature $T_{f}$, independent of the kinetic rate. The differential-diffusion effects, associated with the non-unity value of the fuel Lewis number, change the temperature in the reaction layer from its adiabatic stoichiometric value by a finite amount, which grows when $S$ decreases in dilute systems. The dependences on $S$ and $L_{\mathrm{F}}$ of the B-S flame location $\eta_{f}$, peak temperature $T_{f}$, reactant consumption rates $m_{\mathrm{F}}=m_{\mathrm{O}} / S$, heat-release rate $q m_{\mathrm{F}}$, as well as the fractions $\gamma_{\mathrm{F}}$ and $\gamma_{0}=1-\gamma_{F}$ of the reaction heat that is transported to the fuel and air sides of the flame have been computed for the case of equal feed temperatures; the results, given in Fig. 3, are seen to compare favorably with the analytic predictions when using the approximation $F=\eta$.

The analysis shows how finite-rate effects lead to extinction when the overall reaction has a large value of the nondimensional activation temperature $T_{a} / T_{f}$. In this case small temperature changes, of order $T_{f}^{2} / T_{a}$, associated with small reactant mass 
fractions of order $T_{f} / T_{a}$ in the reaction layer, are enough to affect in a nonlinear way the inner structure of the reaction layer, producing leakage of the reactants. The increasing value of the mass fractions of the reactants, coexisting in the reaction layer, leads to a bending bifurcation of the near B-S solution, at a well-defined extinction Damköhler number, into a second unstable branch of the solution. The analysis of this bending bifurcation has been carried out in Section 4 for large values of the activation energy $T_{a} / T_{f}$ and of the ratio $B / A_{\mathrm{A}}$, after identifying the double limit so as to ensure reactant mass fractions of order $T_{f} / T_{a}$ in the thin reaction layer; this occurs for an effective Damköhler number $\mathcal{D}$, defined in (59), of order unity. The determination of the precise value of $\mathcal{D}$ at extinction requires consideration of the small departures from the equilibrium B-S solution occurring both in the thin reactiondiffusion inner layer and in the much thicker convection-diffusion outer regions, where the corrections, also of order $T_{f} / T_{a}$, are associated with the reactants leaking through the flame.

The asymptotic analysis of the reaction-layer structure leads to a canonical boundary-value problem, identical to that encountered by Liñán [5] for unity values of the Lewis numbers of the reactants. The solution determines the small amount of reactants that leak through the flame in terms of a canonical Damköhler number $\Lambda=\mathcal{D} \exp \left[C_{1}+\gamma_{\mathrm{F}} C_{2}(S+1) / S\right]$, where the proportionality constant corresponds to a shift of the flame-sheet temperature $T_{f}$, by an amount $\left[C_{1}+\gamma_{\mathrm{F}} C_{2}(S+1) / S\right] T_{f}^{2} / T_{a}$, resulting from the feedback influence of the perturbations in the outer regions due to the leakage of the reactants. These perturbations have been calculated using the approximation $F=\eta$, when there are no changes in the excess enthalpy $\xi$ at the flame, and $C_{1}$ is identically zero. The value of $C_{2}$, corresponding to an apparent shift at the flame of the diffusionweighted mixture fraction $\tilde{Z}$, turns out to be moderately small, leading to values of $\Lambda / \mathcal{D}$ that approach $\Lambda / \mathcal{D}=1$ for increasing values of $S$, and are always moderately close to unity, as seen in Fig. 6.

Hence our results indicate that the main effect of differential diffusion on the extinction conditions is associated with the resulting departures of $T_{f}$ from the adiabatic value $T_{5}$, while the corrections to the extinction Damköhler number $D$ arising from the feedback effect of the perturbations in the outer stream on the innerlayer structure are much smaller.

The comparisons of the results of the asymptotic analysis with numerical integrations for moderately large values of the activation energy show satisfactory agreement for values of $S$ of order unity. As expected, errors in the extinction strain rate are of order $\beta^{-1}$, although the proportionality factor involved in the relative errors is somewhat larger than unity, as can be inferred from Fig. 7.

Separate consideration is given to cases with larger values of $S$, or smaller values of $L_{\mathrm{F}}$, leading to flame displacement into the air stream. Under those conditions, flame extinction is seen to occur in a premixed-flame regime, due to the resulting small value of $\gamma_{\mathrm{F}}$, which results in leakage of the fuel, with mass fraction of order $\beta^{-1}$, but negligible leakage of the oxidizer, as discussed in Section 5 , where an analytical approximation of the ratio $\Lambda / \mathcal{D}$ is given.

The asymptotic treatment of diffusion-flame extinction given here is readily applicable to derive predictions of critical strain rates at extinction when the fuel-oxidation chemistry can be described with a one-step reaction with large activation energy, as occurs for instance in lean hydrogen-air mixtures [6], of relevance for non-premixed flames with dilute fuel feed. Model one-step kinetics having an Arrhenius rate with moderately large activation energy has been shown to be sufficiently accurate in numerical descriptions of extinction of methane-air flames [14].

Obviously, the predicted values of the extinction strain rate are dependent on the single-reaction model used, as was clearly shown by Norbert Peters. He proposed an alternative approach [18], based on rate-ratio asymptotics, to generate multistep reduced chemical-kinetic mechanisms for realistic fuels, and for deriving extinction predictions for diffusion flames. The application of this alternative technique helped to clarify the inner structure of hydrocarbon-air diffusion flames, which includes a thin fuel-consumption layer, where the fuel is attacked by radicals-mainly $\mathrm{H}$ and $\mathrm{OH}$-to produce $\mathrm{CO}$ and $\mathrm{H}_{2}$, precluding the leakage of the radicals to the fuel side and the leakage of the fuel to the air side, and an adjacent $\mathrm{H}_{2}$ and $\mathrm{CO}$ oxidation layer, where radical production occurs. This clarification is another example of Peters' numerous visionary contributions to Combustion Science.

\section{Acknowledgments}

The senior author wants to acknowledge the very valuable help of Alain Leppinette in the work that they carried out together on the effects of non-unity Lewis numbers on diffusion-flame extinction. This work was done as part of Alain's PhD requirements and, at the time, could not be finished.

The authors of this paper wish to express their deep gratitude to Norbert Peters for his outstanding contributions to Combustion Science. These include the special role that he played in introducing basic concepts, such as the reduced kinetic mechanisms and the flamelet approach, that could shed the needed light on our understanding of the important engineering application of turbulent combustion.

The senior author of this paper (AL) remembers his first encounter with Norbert Peters, during the 1978 Combustion Meeting in Leeds, as one of the most memorable events in his life. AL was very much impressed by Peters' enthusiastic interest in gaining a deep scientific understanding of the combustion phenomena, using all the techniques available. In Leeds, together with John Clarke, they decided to use the opportunity of the first visit to Madrid of Grisha Sivashinsky, who had initiated his research work in Zel'dovich's combustion group, to organize an informal oneweek encounter in Madrid, including Paul Clavin and Guy Joulin, who were already using Sivashinsky's ideas in their studies of the effects of heat losses in flame instabilities. We had blackboard informal discussions about the effect of strain on flame structures, and about the main difficulties of the description of the kinetics of the combustion reactions, and the role of the disparity of reaction time scales and their strong sensitivity with temperature.

It was due to Norbert Peters' energetic drive that this encounter of Madrid was followed a year later by the first IWOMIC Meeting organized by him in Aachen. This meeting, now truly international with all the groups involved in combustion science, set up a highlevel standard of unrestricted exchange of information among us, leading soon to very fruitful collaborations. Norbert Peters and Forman Williams, with their contagious enthusiasm for science, became role models for these collaborations, leading to the admirable atmosphere of warm friendship among all of us involved in combustion science.

This work was supported by the Spanish MCINN through the CONSOLIDER project \# CSD2010-00010.

\section{References}

[1] N. Peters, Turbulent combustion, Cambridge University Press, Cambridge, U.K., 2000.

[2] N. Peters, Laminar flamelet concepts in turbulent combustion, Prog. Energy Combust. Sci. 10 (1984) 319-339.

[3] F.A. Williams, Recent advances in theoretical descriptions of turbulent diffusion flames, in: S.N.B. Murthy (Ed.). Turbulent Mixing in Non-Reactive and Reactive Flows, Plenum Press, New York (1975), pp. 189-208.

[4] U. Niemann, K. Seshadri, F.A. Williams, Accuracies of laminar counterflow flame experiments, Combust. Flame 162 (2015) 1540-1549. 
[5] A. Liñan, The asymptotic structure of counterflow diffusion flames for large activation energies, Acta Astronaut. 1 (1974) 1007-1039.

[6] D. Fernández-Galisteo, A.L. Sánchez, A. Liñán, F.A. Williams, One-step reduced kinetics for lean hydrogen-air deflagration, Combust. Flame 156 (2009) 985-996.

[7] A. Liñán, M. Vera, A.L. Sánchez, lgnition, liftoff, and extinction of gaseous diffusion flames, Annu, Rev. Fluid Mech. 47 (2015) 293-314.

[8] A. Liñan, The structure of diffusion flames, in: M. Onofri, A. Tesev (Eds.), Fluid Dynamical Aspects of Combustion Theory, Longman Scientific and Technical, Harlow, U.K. (1991), pp. 11-29.

[9] A. Liñan, Diffusion-controlled combustion, in: H. Aref, J.W. Phillips (Eds,), Mechanics for a New Millennium, Kluwer Acad., Dordrecht, Holland (2001), pp. 487-502.

[10] A. Liñan, Lewis number effects on the structure and extinction of diffusion flames due to strain, in: J. Jiménez (Ed.), The Role of Coherent Structures in Modelling Turbulence and Mixing, Springer-Verlag, Berlin (1981), pp. 333 339.

[11] S.H. Chung, C.K. Law, Structure and extinction of convective diffusion flames with general Lewis numbers, Combust. Flame 52 (1983) 59-79.
[12] J.S. Kim, F.A. Williams, Extinction of diffusion flames with nonunity Lewis numbers, J. Eng. Math. 31 (1997) 101-118.

[13] S. Cheatham, M. Matalon, A general asymptotic theory of diffusion flames with application to cellular instability. J. Fluid Mech. 414 (2000) 105-144.

[14] E. Fernández-Tarrazo, A.L. Sánchez, A. Liñán, F.A. Williams, A simple one-step chemistry model for partially premixed hydrocarbon combustion, Combust Flame 147 (2006) 32-38.

[15] F.A. Williams, A review of some theoretical considerations of turbulent flame structure, in: M. Barrère (Ed.). Analytical and Numerical Methods for Investigation of Flow Fields with Chemical Reactions, Especially Related to Combustion, AGARD Conference Proceedings No. 164, AGARD, NATO, Paris (1975), pp. 1-25.

[16] K. Seshadri, N. Peters, F.A. Williams, V. Nayagam, G. Paczko, Asymptotic analysis of quasi-steady n-Heptane droplet combustion supported by cool-flame chemistry, Combust. Th. Modelling (2016). (To appear)

[17] M. Nishioka, C.K. Law, T. Takeno, A flame-controlling continuation method for generating s-curve responses with detailed chemistry, Combust. Flame 104 (1996) 328-342.

[18] K. Seshadri, N. Peters, Asymptotic structure and extinction of methane-air diffusion flames, Combust. Flame 73 (1988) 23-44. 